\title{
INTERACTION OF MASSIVE BLACK HOLE BINARIES WITH THEIR STELLAR ENVIRONMENT. III. SCATTERING OF BOUND STARS
}

\author{
Alberto Sesana, ${ }^{1}$ Francesco HaArdt, ${ }^{1,2}$ and Piero Madau ${ }^{3}$ \\ Received 2007 October 18; accepted 2008 May 31
}

\begin{abstract}
We develop a formalism for studying the dynamics of massive black hole binaries embedded in gravitationally bound stellar cusps, and study the binary orbital decay by three-body interactions, the impact of stellar slingshots on the density profile of the inner cusp, and the properties of the ejected hypervelocity stars (HVSs). We find that the scattering of bound stars shrinks the binary orbit and increases its eccentricity more effectively than that of unbound ambient stars. Binaries with initial eccentricities $e \gtrsim 0.3$ and/or unequal-mass companions $\left(M_{2} / M_{1} \lesssim 0.1\right)$ can decay by three-body interactions to the gravitational wave emission regime in less than a Hubble time. The stellar cusp is significantly eroded, and cores as shallow as $\rho \propto r^{-0.7}$ may develop from a preexisting singular isothermal density profile. A population of HVSs is ejected in the host galaxy halo, with a total mass $\sim M_{2}$. We scale our results to the scattering of stars bound to $\mathrm{Sgr} \mathrm{A}^{*}$, the massive black hole in the Galactic center, by an inspiraling companion of intermediate mass. Depending on binary mass ratio, eccentricity, and initial slope of the stellar cusp, a core of radius $\sim 0.1 \mathrm{pc}$ typically forms in 1-10 Myr. On this timescale about 500-2500 HVSs are expelled with speeds sufficiently large to escape the gravitational potential of the Milky Way.
\end{abstract}

Subject headings: black hole physics — methods: numerical — stellar dynamics

Online material: color figures

\section{INTRODUCTION}

In the standard paradigm of cosmic structure formation, it is expected that many wide massive black hole binaries (MBHBs) will form following the mergers of two massive galaxies (e.g., Begelman et al. 1980; Volonteri et al. 2003a; Mayer et al. 2007). The binary will subsequently shrink due to stellar or gas dynamical processes and may ultimately coalesce by emitting a burst of gravitational waves. It was first proposed by Ebisuzaki et al. (1991) that the heating of the surrounding stars by a decaying massive black hole (MBH) pair would create a lowdensity core out of a preexisting cuspy (e.g., $\rho \propto r^{-2}$ ) stellar profile. In a purely stellar background a "hard" binary shrinks by capturing the stars that pass close to the holes and ejecting them at much higher velocities, a superelastic scattering process ("gravitational slingshot") that depletes the nuclear region. Observationally, there is evidence in early-type galaxies for a systematically different distribution of surface brightness profiles, with faint ellipticals showing steep power-law profiles (cusps), while bright ellipticals have much shallower stellar cores (e.g., Faber et al. 1997; Ravindranath et al. 2001). Dwarf ellipticals seem to elude this paradigm by showing somewhat flat profiles, similar to bright ones (Graham \& Guzmán 2003). Latetype spirals tend to show steep central cusps, as in the case of the Milky Way or Andromeda. Detailed $N$-body simulations have confirmed the cusp-disruption effect of a hardening MBHB (Makino \& Ebisuzaki 1996; Quinlan \& Hernquist 1997; Milosavljevic \& Merritt 2001), while semianalytic modeling in the framework of hierarchical structure formation theories has shown that the cumulative damage done to stellar cusps by decaying black hole pairs may explain the observed correlation between the "mass

\footnotetext{
1 Dipartimento di Scienze, Università dell'Insubria, via Valleggio 11, 22100 Como, Italy.

2 Istituto Nazionale di Fisica Nucleare (INFN), Rome, Italy.

3 Department of Astronomy and Astrophysics, University of California, 1156 High Street, Santa Cruz, CA 95064.
}

deficit" (the mass needed to bring a flat inner density profile to a $r^{-2}$ cusp) and the mass of the nuclear black hole (Volonteri et al. 2003b).

This is the third paper of a series aimed at the detailed study of the interaction between MBHBs and their stellar environment. In Sesana et al. (2006, 2007a, hereafter Paper I and Paper II, respectively), we analyzed the three-body scatterings between a MBHB and background stars unbound to the binary. The assumption of a fixed background breaks down once the binary has ejected most of the stars on intersecting orbits, and the extraction of energy and angular momentum from the binary can continue only if new stars can diffuse into low angular momentum orbits (refilling the binary's phase-space "loss cone"), or via gas processes. In galaxies with inner cores or shallow cusps, only a small fraction of the loss cone is confined within the sphere of influence of the binary, and the approximation of a background of unbound stars is reasonable. A similar argument holds also in the case of a galaxy with a steep cusp hosting a nearly equal mass binary $\left(M_{1} \sim M_{2}\right)$. The radius of influence of such a pair, $r_{\text {inf }}=G\left(M_{1}+M_{2}\right) /\left(2 \sigma^{2}\right)$, where $\sigma$ is the stellar velocity dispersion, is of the order of the binary hardening radius, $a_{h}=$ $G M_{2} / 4 \sigma^{2}$, and only few low angular momentum stars have orbits with semimajor axis $\leqslant r_{\text {inf }}$. This is not true for unequal-mass binaries, where $r_{\text {inf }} \gg a_{h}$, and almost all interacting stars are bound to $M_{1}$.

In this paper we develop a formalism for studying the dynamics of MBHBs embedded in gravitationally bound stellar cusps. The plan is as follows. In $\S 2$ we describe our suite of three-body scattering experiments between the black hole pair and ambient bound stars. Our numerical results are used in $\S \S 3$ and 4 to construct a "hybrid model" of binary dynamics and investigate the orbital decay and shrinking of MBHBs in timeevolving stellar cusps. The properties of the ejected HVSs are discussed in $\S 5$. The massive black hole $\left(\mathrm{Sgr} \mathrm{A}^{*}\right)$ in the Galactic center and the stars around it offer a unique opportunity to study stellar dynamics in the extreme environment around a 


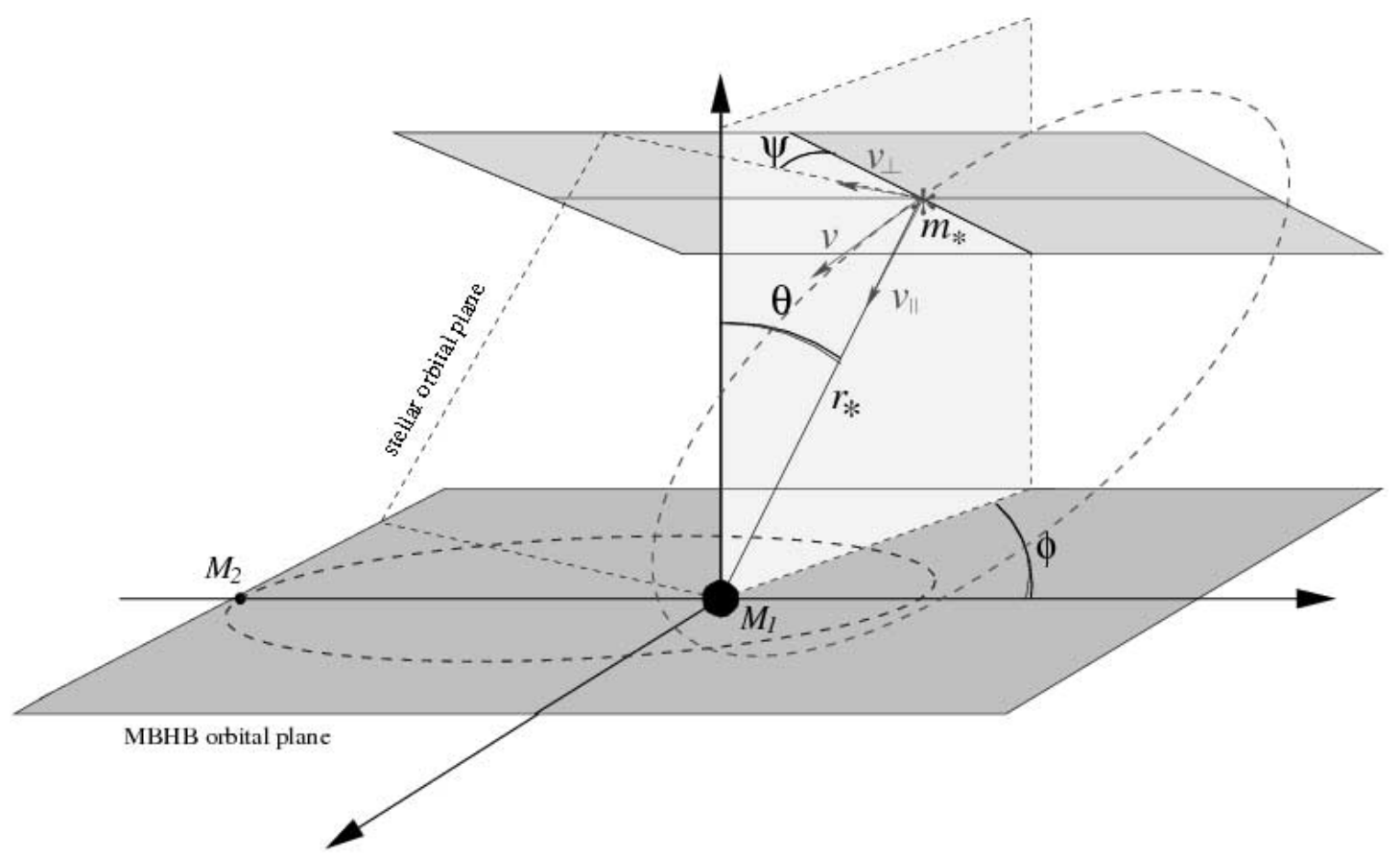

FIG. 1.-Geometry of the three-body scattering experiments. The stellar orbit is determined by $E_{*}$ and $L_{*}$, while its orientation with respect to the binary plane is determined by angle $\psi$. See the text for a definition of all symbols. [See the electronic edition of the Journal for a color version of this figure.]

relativistic potential. The scattering of stars bound to Sgr A* by an inspiraling intermediate-mass black hole (IMBH) is treated in $\S 6$. Finally, we present a brief summary in $\S 7$.

\section{SCATTERING EXPERIMENTS WITH BOUND STARS}

Consider a massive black hole of mass $M_{1}$ surrounded by a stellar cusp (with density profile $\rho \propto r^{-\gamma}, \gamma>0$ ), interacting with a secondary "intruder" hole of mass $M_{2}<M_{1}$. As the binary separation decays, the effectiveness of dynamical friction slowly declines because distant stars perturb the binary's center of mass but not its semimajor axis. The bound pair then loses orbital energy by capturing ambient stars and ejecting them at much higher velocities, a three-body scattering process known as the "gravitational slingshot." For unequal-mass binaries (mass ratio $q \lesssim 0.1), a_{h} \ll r_{\text {inf }}$, implying that the stellar mass inside $a_{h}$ is $M_{\text {cusp }}\left(<a_{h}\right) \ll M_{1}$. In this case, the contribution to the potential energy given by the stellar distribution during a binary-star interaction can be ignored, and the problem can be tackled by means of three-body scattering experiments. Following the guidelines fully described in Paper I, we performed numerical experiments to study the interaction of a MBHB with a star of mass $m_{*}$ $\left(m_{*} \ll M_{2}<M_{1}\right)$ bound to $M_{1}$. Note that for extreme mass ratios $q \ll 1$, the encounter is essentially a two-body scattering as the star and the secondary hole move in the static potential of the primary.

\subsection{Initial Conditions and Orbit Integration}

The integration of the three-body encounter equations is greatly simplified by setting the center of mass of the binary at rest in the origin of the coordinate system. The binary orbits counterclockwise in the $(x, y)$-plane, and the apastron of $M_{2}$ is located along the positive $x$-axis. Stars are drawn from a spherical isotropic distribution bound to $M_{1}$. The problem is completely defined by 10 variables:

1. the binary mass ratio $q \equiv M_{2} / M_{1}$;

2. the binary eccentricity $e$;

3. the stellar mass $m_{*}$;
4. the initial distance between the primary hole and the star, $r_{*} \equiv\left|\boldsymbol{r}_{*}\right|$

5. the specific energy of the stellar orbit around $M_{1}, E_{*}=$ $-G M_{1} /\left(2 a_{*}\right)$, or equivalently the semimajor axis of the stellar orbit, $a_{*}$;

6. the specific angular momentum of the stellar orbit $L_{*}=$ $\left|\boldsymbol{v} \times \boldsymbol{r}_{*}\right|$, where $v \equiv|\boldsymbol{v}|$ is the stellar velocity relative to $M_{1}$;

7. four angles: $\theta$ and $\phi$ describing the latitude and longitude of the star, $\psi$ defining the orientation of $v_{\perp}$ (the stellar velocity component normal to $\boldsymbol{r}_{*}$ ), i.e., selecting the orbital plane of the star, and $\Psi$, the initial binary phase.

Compared to the unbound scattering problem (which is defined by nine variables; see Paper I), there is here the extra variable $r_{*}$, and $E_{*}$ and $L_{*}$ replace the asymptotic initial speed and impact parameter of the incoming field star. A sketch of the experiment setup is given in Figure 1. In each numerical integration, the binary mass $M=M_{1}+M_{2}$ and separation $a$ are set equal to unity and the stellar mass to $m_{*}=10^{-6} \mathrm{M}$. Scattering events are simulated in sets of $5 \times 10^{4}$ trials for fixed values of $q$ and $e$. We sample five values of the binary eccentricity, $e=0,0.1,0.3,0.6$, and 0.9 , and five values of the binary mass ratio, $q=1 / 9,1 / 27$, $1 / 81,1 / 243$, and $1 / 729$, for a grand total of 25 models. The distribution of stars bound to $M_{1}$ is modeled as follows. The stellar semimajor axis $a_{*}$ is randomly sampled from 50 logarithmic bins spanning the range $0.03 a<a_{*}<10 a$. The angular momentum is sampled in the interval $\left[0, L_{*, \max }^{2}\right]$ according to an equal probability distribution in $L_{*}^{2}$, where $L_{*, \max }^{2}=G M_{1} a_{*}$ is the specific angular momentum of a circular orbit of radius $a_{*}$. A population of stars with such distribution in $L_{*}^{2}$ has mean eccentricity $\left\langle e_{*}\right\rangle=0.66$, corresponding to $\left\langle v_{\perp}\right\rangle=2\left\langle v_{\|}\right\rangle$. This condition defines an isotropic stellar distribution (e.g., Quinlan et al. 1995).

The quantities $E_{*}$ and $L_{*}$ define the shape of the stellar orbit. We sample the initial value of $r_{*}$ from the distribution $\mathcal{P}\left(r_{*}\right) d r_{*}$,

$$
\mathcal{P}\left(r_{*}\right) d r_{*}=\frac{2\left|E_{*}\right|^{3 / 2}}{\pi G M_{1} \sqrt{E_{*}-L_{*}^{2} / 2 r_{*}^{2}+G M_{1} / r_{*}}} d r_{*},
$$




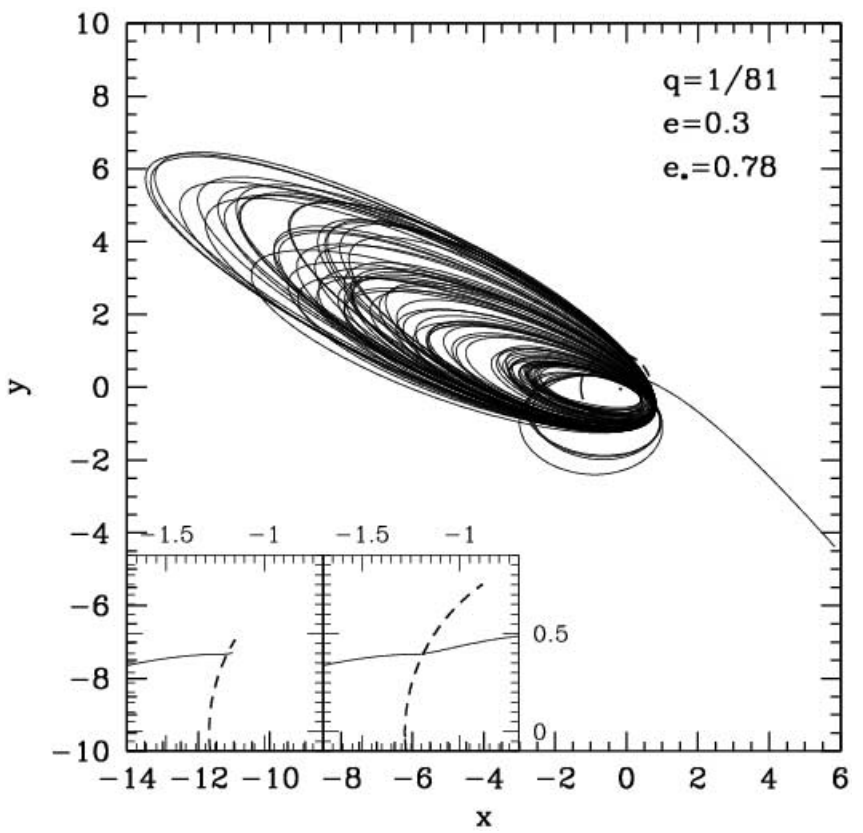

FIG. 2.-Example of a strong three-body interaction unbinding the star. The parameters of the system are listed in the figure. The star is ejected toward the lower right with a kick velocity $V \approx 0.05 V_{c}$. The thick circle marks the orbit of $M_{2}$, while the small central dot marks the location of $M_{1}$. The insets zoom in at the moment of the scattering between the star (approaching from the left) and $M_{2}$ (approaching from below). [See the electronic edition of the Journal for a color version of this figure.]

which is proportional to the fraction of time spent by the star at a distance between $r_{*}$ and $r_{*}+d r_{*}$ from $M_{1}$. The probability $\mathcal{P}\left(r_{*}\right)$ is defined in the range $r_{*,-}<r_{*}<r_{*,+}$, where

$$
\begin{aligned}
& r_{*,-}=L_{*}^{2}\left(G M_{1}+\sqrt{G^{2} M_{1}^{2}+2 E_{*} L_{*}^{2}}\right)^{-1}, \\
& r_{*,+}=L_{*}^{2}\left(G M_{1}-\sqrt{G^{2} M_{1}^{2}+2 E_{*} L_{*}^{2}}\right)^{-1} .
\end{aligned}
$$

The angles $\theta$ and $\phi$ are randomly generated to reproduce a uniform density distribution over a spherical surface centered on $M_{1}$, while the orientation angle $\psi$ is chosen from a uniform distribution in the range $[0,2 \pi]$. We start numerical integration with $M_{2}$ at its apastron $(\Psi=0)$ and have checked that the chosen initial phase of the binary does not affect our results. The orbit of the pair is in the $(x, y)$-plane with the center of mass at coordinates $(0,0,0)$.

The nine coupled, second-order, differential equations of the three-body problem are integrated using the most recent version of the subroutine DOPRI5, based on an explicit Runge-Kutta method of the order of 4(5) taken from Dormand \& Prince (1978). A complete description of the integrator can be found in Hairer et al. (1993). The integration is stopped if any of these events occurs:

1. The star leaves the sphere of radius $r=\left(10^{10} \mu / M\right)^{1 / 4} a_{0}$ with positive total energy, where $\mu=M_{1} M_{2} / M$ is the reduced mass of the binary and $a_{0}$ its initial semimajor axis. At $r$ the force induced by the quadrupole moment of the binary is 10 orders of magnitude smaller than the total force acting on the star at a distance $a_{0}$.

2. The physical integration time exceeds 1 Gyr.

3. The integration reaches $10^{8}$ time steps. This typically corresponds to the complete integration of $10^{4}-10^{5}$ binary orbits.

Examples of integrated stellar orbits are given in Figures 2 and 3. Figure 2 shows a strong interaction: the star has a close

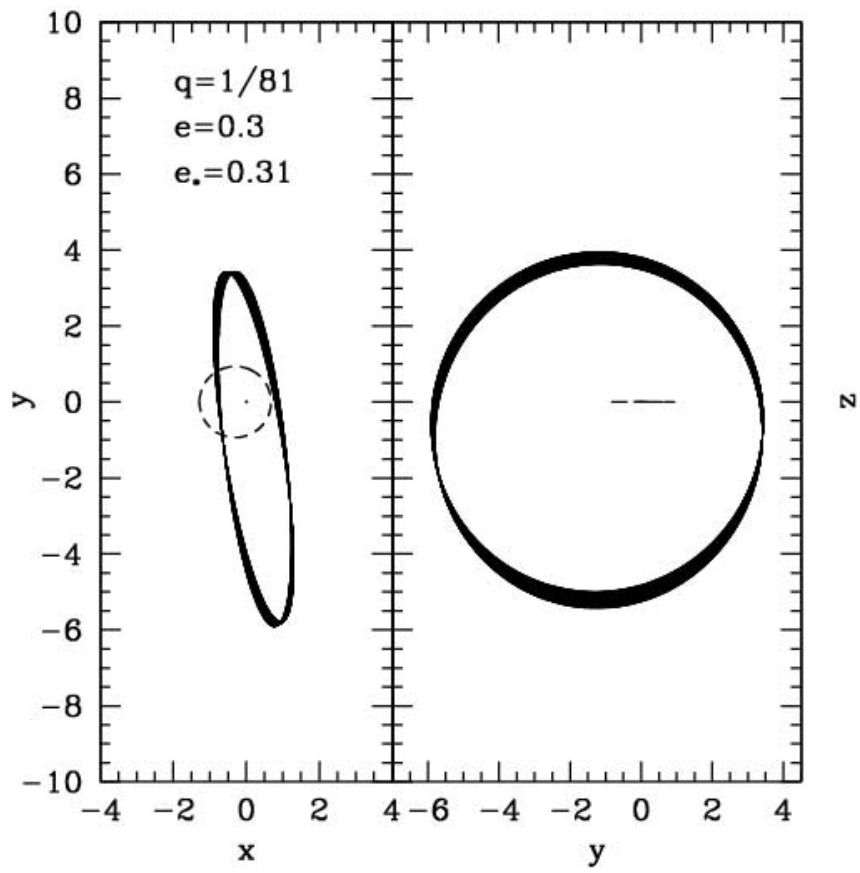

FIG. 3.-Example of a weak three-body interaction. The thick circle marks the orbit of $M_{2}$, while the small central dot marks the location of $M_{1}$. Left: Projection onto the binary $(x, y)$-plane. Right: Projection onto the $(y, z)$-plane. [See the electronic edition of the Journal for a color version of this figure.]

encounter with $M_{2}$ and leaves the binary sphere of influence with positive energy. Depending on the value of $a_{*}$ we have a variable number of numerically "abandoned events," i.e., bound triple systems that do not readily produce an ejection. Figure 3 shows one such event: the periastron of the star is much larger than the binary orbital separation $a$, and the star is not perturbed by the binary quadrupole field, except for the precession of its periastron.

\subsection{Tests}

We have performed a number of tests to check the sensitivity of our results on numerics. Because of the intrinsically chaotic nature of the three-body problem, the properties of the ejected stars are meaningful only for a statistically significant sample. The integration of the full three-body problem allows us to directly control the conservation of total energy and angular momentum. The code adjusts the integration step size to keep the fractional error per step in position and velocity, $\epsilon$, below $10^{-13}$. This allows a total energy conservation accuracy of $\Delta E / E \sim 10^{-9}$ in a single orbit integration; i.e., for $m_{*} / M \simeq 10^{-6}$ the energy of the star is conserved at the level of one part in a thousand during a single orbit. We varied $\epsilon$ between $10^{-11}$ and $10^{-15}$ and $m_{*} / M$ between $10^{-5}$ and $10^{-7}$, and found no significant differences in the statistics of the ejected population. We have also checked that the mean energy and angular momentum exchanges scale linearly with $m_{*}$. A set of longer orbit integrations was performed to test that the number of abandoned events does not depend on the finite numbers of time steps allowed, again finding no systematic decrease in such a number.

\subsection{Outputs}

Each logarithmic bin in $a_{*}$ was sampled by $10^{3}$ stars. We calculated the mean energy and angular momentum exchange between the MBHB and the stars, the final velocity and angular distribution of the scattered stars, the fraction $f_{\text {ej }}$ of stars that are ejected in the interaction, and the ejection timescales. The fraction $f_{\mathrm{ej}}$ is plotted versus $a_{*} / a$ in Figure 4 for different values of the binary 


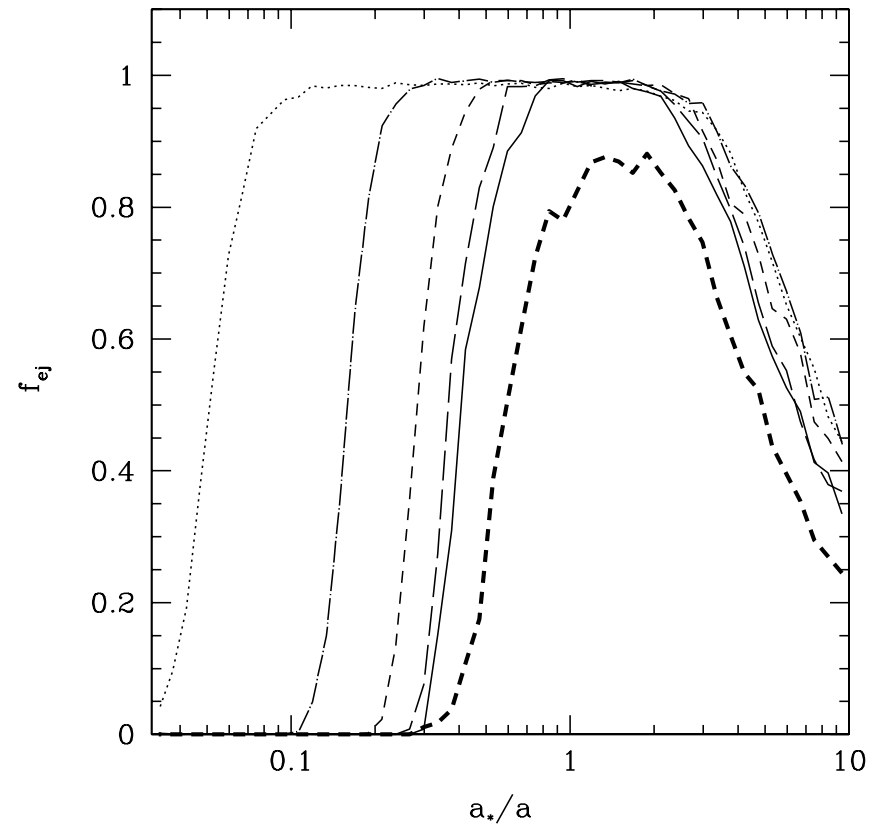

FIG. 4.-Fraction of stars ejected in the interaction as a function of $a_{*} / a$. Thin curves are for $q=1 / 9$ and different eccentricities: $e=0$ (solid line), $e=0.1$ (longdashed line), $e=0.3$ (short-dashed line), $e=0.6$ (dot-dashed line), and $e=0.9$ (dotted line). The thick dashed line is for $q=1 / 729$ and $e=0.3$.

parameters $q$ and $e$. Eccentric binaries can eject stars that are initially very tightly bound to $M_{1}$, i.e., with $a_{*}$ as small as $0.1 a$. For $a_{*} \gtrsim 3 a$, the expelled fraction declines significantly, regardless of binary eccentricity. It is also evident that the ejection process is more efficient for large values of $q$.

Figure 5 shows the mean fractional eccentricity change of the pair after each scattering, $\langle\Delta e / e\rangle$, as a function of $a_{*} / a$. This quantity is found to scale linearly with $m_{*} / M$. Stars with $a_{*}<a$ typi-

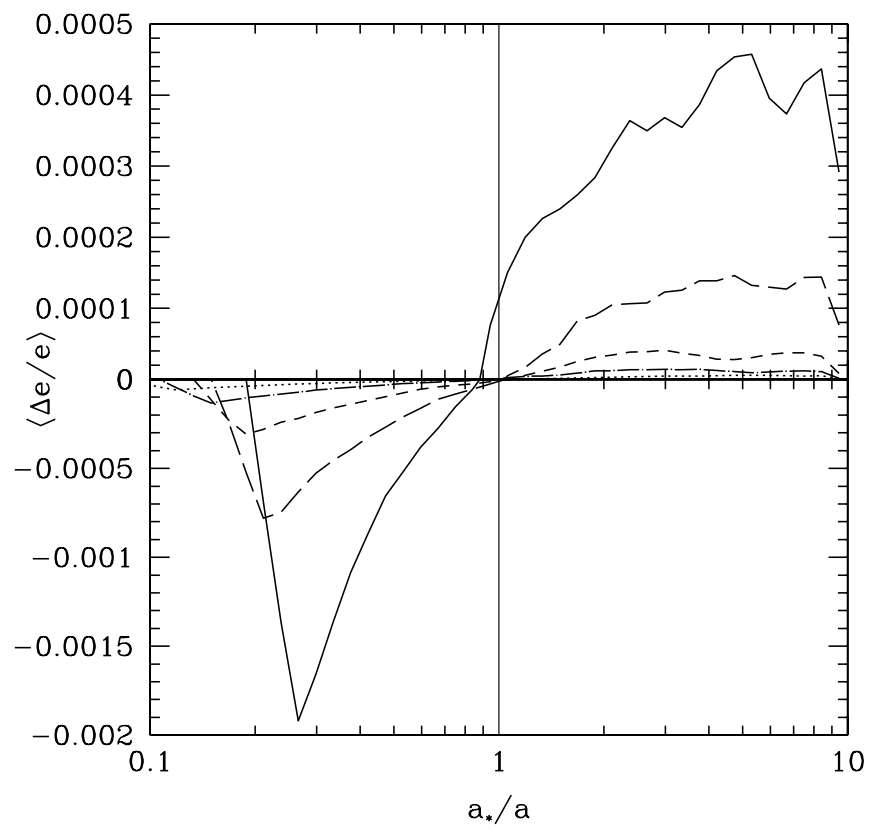

FIG. 5.-Mean fractional eccentricity change, $\langle\Delta e / e\rangle$, of the binary after a scattering, as a function of $a_{*} / a$. The assumed initial eccentricity is 0.6 , and the different curves are for $q=1 / 729$ (solid line), $q=1 / 243$ (long-dashed line), $q=1 / 81$ (short-dashed line), $q=1 / 27$ (dot-dashed line), and $q=1 / 9$ (dotted line). Note the different scales of the positive and negative $y$-axis.

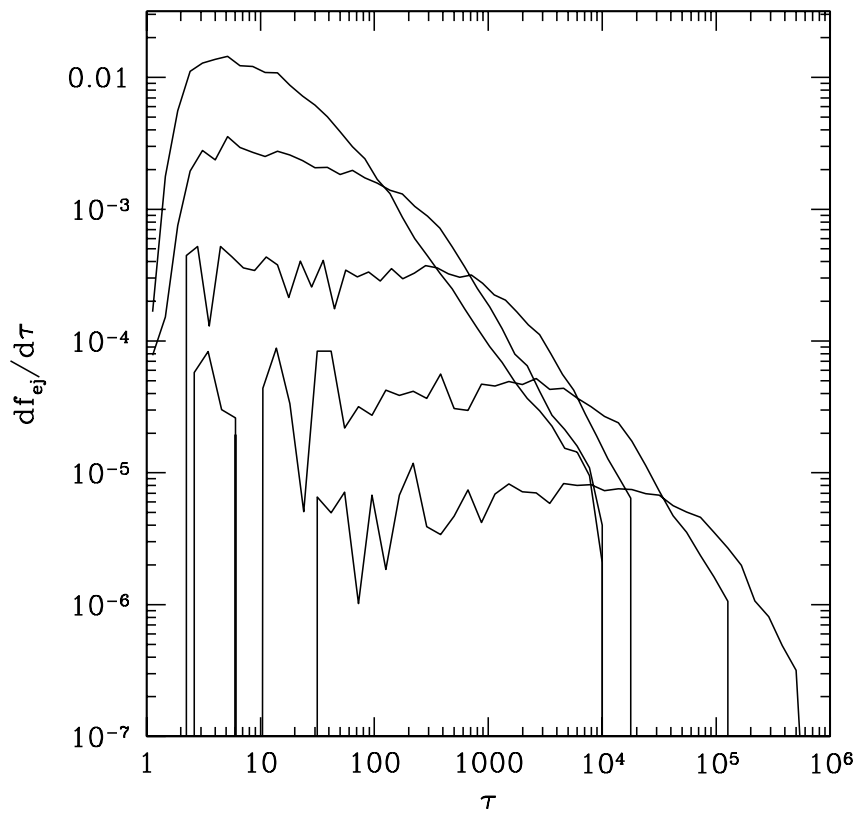

FIG. 6.-Differential distribution of ejection timescales $\tau$. The assumed initial eccentricity is 0.3 , and the different curves, from top to bottom, are for $q=1 / 9$, $1 / 27,1 / 81,1 / 243$, and $1 / 729$.

cally tend to reduce the binary eccentricity, while stars with $a_{*}>a$ work in the opposite direction. An eccentric binary spends most of its period near its apocenter, so in the case $a_{*}>a$ the probability of a close star-binary encounter (and subsequent star ejection) is maximal at binary apocenter. In the instantaneous interaction the binary velocity decreases. As the binary velocity is nearly tangential close to the apocenter, the binary is forced on a more radial orbit. On the contrary, a star with $a_{*}<a$ "feels" the secondary hole $M_{2}$ when this approaches the primary $M_{1}$ at a distance $\sim a_{*}$. At that point, the interaction with the star is unlikely to occur close to the pericenter of $M_{2}$ (where the time spent by $M_{2}$ at the pericenter is very small), and typically also extracts a large radial component from the velocity of the secondary black hole, hence causing circularization. Note that in this case the absolute value of $\Delta e$ is larger, according to the $a / a_{*}$ dependence in equation (9) (see the different positive and negative $y$-axis scales in Fig. 5). We will return on this point in $\S 3$, giving a simple, physically motivated, mathematical derivation of this qualitative argument.

Figure 6 depicts the fractional number of stars ejected in the time interval $\tau$ and $\tau+d \tau$ as a function of the ejection timescale $\tau$. The latter is measured in units of the binary orbital period at separation $a_{0} \equiv a(t=0), P_{0}=2 \pi\left[a_{0}^{3} /(G M)\right]^{1 / 2}$, and is defined as the time elapsed from the start of numerical integration to the moment the interacting star reaches, with positive energy, a distance $\gg a$ from the binary center of mass. For large values of $q$, it typically takes the MBHB just a few orbits to expel the star, while lowering $q$ makes the distribution of slingshot timescales broader and flatter. The ejection rate remains approximately constant, or decreases slowly, for $\tau \lesssim 5 / q^{2}$, and drops dramatically afterward. The binary eccentricity plays no role in all the tested cases. Figure 7 shows the mean ejection timescale [i.e., the integral of $\tau\left(d f_{\mathrm{ej}} / d \tau\right)$ in $\left.d \tau\right]$ as a function of the star eccentricity $e_{*}$, for different values of $q$ and $e$. As a first approximation, one can assume that the cusp remains isotropic during the binary orbital evolution.

We checked that our results are practically the same considering $5 a$ rather than $10 a$ as the outer boundary in the sampled $a_{*}$ distribution. This is because the binary-stars interaction is dominated, 


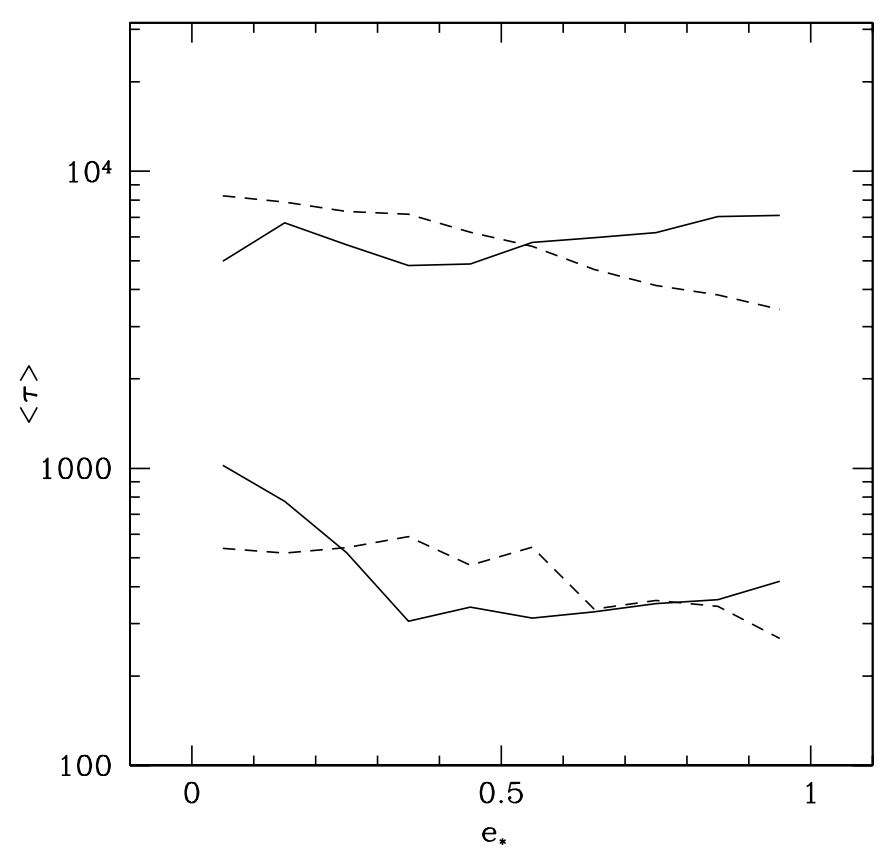

FIG. 7.- Mean ejection timescale $\langle\tau\rangle$ of stars as a function of the star eccentricity $e_{*}$, assuming a MBHB with $e=0.1$ (solid lines) and $e=0.9$ (dashed lines). The two upper (lower) curves are for $q=1 / 81(q=1 / 9)$.

in terms of energy and angular momentum exchange, by those stars whose $a_{*}$ is $\sim a$.

From our scattering experiments, we can finally derive the bivariate distribution functions $h_{1}\left(V, \theta \mid a_{*}\right)$ and $h_{1}\left(V, \phi \mid a_{*}\right)$, along the same line described in Paper II. For a given $a_{*}$, we record the number of stars with ejection speed in the interval $V, V+d V$, leaving the binary with latitude (longitude) at infinity in the interval $\theta, \theta+d \theta(\phi, \phi+d \phi)$. The differential distributions are normalized as follows:

$$
\int_{0}^{\infty} d V \int_{0}^{\pi} d \theta h_{1}\left(V, \theta \mid a_{*}\right)=\int_{0}^{\infty} d V \int_{0}^{2 \pi} d \phi h_{1}\left(V, \phi \mid a_{*}\right)=1 .
$$

The subscript " 1 " is meant to indicate that the scattering experiments are performed for a binary at separation $a=1$.

\section{HYBRID MODEL OF BINARY DYNAMICS}

To study the impact of the gravitational slingshot on the dynamical evolution of a MBHB, we have developed a self-consistent hybrid model, in which numerical results of scattering experiments in a fixed stellar background are coupled to an analytical formulation of loss-cone depletion. This technique allows us to simultaneously follow the orbital decay of the pair as well as the time evolution of the stellar cusp. The hybrid model is similar, in spirit, to the case extensively discussed in Paper II of an unbound stellar population interacting with a black hole pair. Scattering experiments are performed for a binary at fixed orbital separation $a$, and the results are scaled to any value of $a$. Then by specifying the pace at which $a$ evolves, we can solve for the binary orbital decay with time. Mathematical details, however, are different from the scheme developed in Paper II. In the unbound case, the pair interacts with a stellar population approaching its sphere of influence at a given rate, and the typical three-body interaction is a "fast" process. For stars bound to $M_{1}$ instead, the interacting stellar population is in place from the beginning. The temporal evolution of the system is then determined by the ejection timescale rather than by the supply rate.

It is convenient to describe the stellar cusp using $d N_{*} / d a_{*}$, the differential number of stars orbiting $M_{1}$ with semimajor axis in the range $a_{*}, a_{*}+d a_{*}$. For an isotropic stellar distribution

$$
\frac{d N_{*}}{d a_{*}}=C 4 \pi a_{*}^{2} \rho\left(r=a_{*}\right)
$$

where the stellar density profile is

$$
\rho(r)=\rho_{0}\left(\frac{r}{r_{0}}\right)^{-\gamma}
$$

and $C$ is a fudge factor that depends on the cusp slope, $C=$ $(1.013,0.872,0.831)$ for $\gamma=(2,1.75,1.5)$ (Ivanov et al. 2005). Two differential equations determine the rate of change of orbital separation and eccentricity:

$$
\begin{gathered}
\frac{d a}{d t}=-\frac{2 a^{2}}{G M_{1} M_{2}} \int_{0}^{\infty} \Delta \mathcal{E} \frac{d^{2} N_{\mathrm{ej}}}{d a_{*} d t} d a_{*}, \\
\frac{d e}{d t}=\int_{0}^{\infty} \Delta e \frac{d^{2} N_{\mathrm{ej}}}{d a_{*} d t} d a_{*} .
\end{gathered}
$$

We start numerical integration at time $t=0$, orbital separation $a_{0}$, and binary eccentricity $e_{0}$. The terms $\Delta e, \Delta \mathcal{E}$, and $d^{2} N_{\text {ej }} / d a_{*} d t$ are measured from our scattering experiments.

The scaling of the eccentricity change with binary parameters $m_{*} / M, e, q$, and $a / a_{*}$ can be derived from (Quinlan 1996)

$$
\Delta e=-\frac{\left(1-e^{2}\right)}{2 e}\left(\frac{\Delta \mathcal{E}}{\mathcal{E}}+\frac{2 \Delta \mathcal{L}_{z}}{\mathcal{L}_{z}}\right)
$$

where $\mathcal{L}_{z}=\mu\left[G M a\left(1-e^{2}\right)\right]^{1 / 2}$ and $\mathcal{E}=-G M_{1} M_{2} /(2 a)$ are the total angular momentum and energy of the binary, respectively. From $\Delta \mathcal{E}=-\Delta \mathcal{E}_{*} \sim-G M_{1} m_{*} /\left(2 a_{*}\right)$ (in a typical encounter, on average, the star gets a kick $\sim V_{c}$ ) and $\Delta \mathcal{L}_{z}=-\Delta \mathcal{L}_{*} \sim-\mathcal{L}_{*}=$ $-m_{*}\left[G M a_{*}\left(1-e_{*}^{2}\right)\right]^{1 / 2}$, assuming $M_{1} \simeq M$, we have

$$
\Delta e \frac{M}{m_{*}} \approx \frac{\left(1-e^{2}\right)}{2 e q}\left[\sqrt{\frac{\left(1-e_{*}^{2}\right) a_{*}}{\left(1-e^{2}\right) a}}-\frac{a}{a_{*}}\right] .
$$

This confirms the qualitative argument developed in $\S 2.3$ and accounts for the features shown in Figure 5: the mean $\Delta e$ scales with the inverse of the mass ratio, and from $\left\langle e_{*}\right\rangle \simeq 0.67$, results are negative for $a_{*} / a \lesssim\left(1-e^{2}\right)^{1 / 3}$ (promoting circularization) and positive for larger $a_{*} / a$ (hence increasing the binary eccentricity).

The binary energy change per scattering, $\Delta \mathcal{E}$, is related to the change of stellar energy $\Delta \mathcal{E}_{*}$ by $\Delta \mathcal{E}_{*} \simeq-\Delta \mathcal{E}$, where we have neglected the energy change of the binary center of mass (which is a factor of $m_{*} / M$ smaller than $\Delta \mathcal{E}$ ). The quantity $\Delta \mathcal{E}_{*}$ can be written as

$$
\Delta \mathcal{E}_{*}\left(a_{*}, a, e\right)=\frac{G M_{1} m_{*}}{2 a_{*}}+\frac{1}{2} k m_{*} V_{c}^{2}(a)
$$

where $V_{c}(a)=(G M / a)^{1 / 2}$ is the circular velocity of the binary. The numerical factor $k$ depends on the ratio $a_{*} / a$ and on $e$, and is derived from our numerical experiments. 
The term $d^{2} N_{\mathrm{ej}} / d a_{*} d t$ quantifies the number of stars orbiting $M_{1}$ within $a_{*}$ and $a_{*}+d a_{*}$ that are ejected from the system in the time interval between $t$ and $t+d t$. This term depends on $a_{*}, a, e$, and $t$ and is determined as follows. From scattering experiments we derive the distribution $P(\tau, s \mid e) d \tau$ describing the probability that a star at $s \equiv a_{*} / a$ becomes unbound from a binary with eccentricity $e$ in the time interval $(\tau, \tau+d \tau)$. Note that the distribution of ejection timescales plotted in Figure 6 is simply the probability function $P$ averaged over $s$,

$$
\frac{d f_{\mathrm{ej}}}{d \tau}=\frac{\int P(\tau, s \mid e) d s}{\int d s} .
$$

When $s \ll 1$ (the exact value depending on $q$ and $e$ ) most stars remain bound to $M_{1}$, and $P=0$ (see Fig. 4). As $s \rightarrow 1$ all interacting stars are instead expelled. We can see from Figure 6 that in the ejection regime the distribution $P$ is nearly independent on $s$ and has the same functional form (as a function of $\tau$ ) of $d f_{\mathrm{ej}} / d \tau$. From $P$ we can then compute $d^{2} N_{\mathrm{ej}} / d a_{*} d t$ as a function of $t$ if we set $\tau=\left(a_{0} / a\right)^{3 / 2} t$, where the term $\left(a_{0} / a\right)^{3 / 2}$ accounts for the change in the time units of $P$ as $a$ changes. Although not formally correct, the scheme catches the basic physics of the interaction in a timedependent fashion.

Suppose now that in a small time interval $\Delta t_{0}$ after the beginning of the interaction the binary remains at constant separation $a_{0}$ corresponding to $s_{0} \equiv a_{*} / a_{0}$. The number of stars (with semimajor axis in the interval $a_{*}, a_{*}+d a_{*}$ ) ejected in such time interval is

$$
\mathcal{F}_{0} \equiv \frac{d^{2} N_{\mathrm{ej}}}{d a_{*} d t}(0) \Delta t_{0}=\frac{d N_{*}}{d a_{*}} P\left(0, s_{0}\right) \Delta t_{0}
$$

where for simplicity we have omitted the dependence of $P$ on the eccentricity. After $\Delta t_{0}$ and for an interval $\Delta t_{1}$, the binary settles to a new separation $a_{1}$, corresponding to $s_{1} \equiv a_{*} / a_{1}$. The number of stars ejected in the time interval $\Delta t_{0}, \Delta t_{0}+\Delta t_{1}$ is

$$
\mathcal{F}_{1}=\left(\frac{d N_{*}}{d a_{*}}-\mathcal{F}_{0}\right) \frac{P\left(\Delta t_{0}, s_{1}\right)}{\int_{\Delta t_{0}}^{\infty} P\left(t^{\prime}, s_{1}\right) d t^{\prime}} \Delta t_{1}\left(\frac{a_{0}}{a_{1}}\right)^{3 / 2} .
$$

The integral in the denominator of the right-hand side renormalizes the distribution $P$ in the time interval $\left[\Delta t_{0}, \infty\right]$ so that the correct number of stars is involved. Iterating we have

$$
\mathcal{F}_{j}=\left(\frac{d N_{*}}{d a_{*}}-\sum_{i=0}^{j-1} \mathcal{F}_{i}\right) \frac{P\left(t, s_{j}\right)}{\int_{t}^{\infty} P\left(t^{\prime}, s_{j}\right) d t^{\prime}} \Delta t_{j}\left(\frac{a_{0}}{a_{j}}\right)^{3 / 2},
$$

where $t \equiv \sum_{i=0}^{j-1} \Delta t_{i}$. In differential form,

$$
\frac{d^{2} N_{\mathrm{ej}}}{d a_{*} d t}(t)=\left(\frac{d N_{*}}{d a_{*}}-\int_{0}^{t} \frac{d^{2} N_{\mathrm{ej}}}{d a_{*} d t^{\prime}} d t^{\prime}\right) \frac{P(t, s)}{\int_{t}^{\infty} P\left(t^{\prime}, s\right) d t^{\prime}}\left[\frac{a_{0}}{a(t)}\right]^{3 / 2},
$$

where $s=s(t)=a_{*} / a(t)$. Simultaneous numerical integration of the three coupled equations (6), (7), and (15) self-consistently solves for the evolution of the binary and the depletion of the stellar cusp. The integration is performed using the subroutine DOPRI5. The time step is adapted in order to keep the fractional error per step $\leq 10^{-10}$, a value much lower than the error associated with the employed linear interpolation of $\Delta e$ and $\Delta \mathcal{E}$.

The bivariate distributions $h_{1}\left(V, \theta \mid a_{*}\right)$ and $h_{1}\left(V, \phi \mid a_{*}\right)$ derived from our suite of scattering experiments (see $\S 2$ ) can be convolved with the ejection rate $d^{2} N_{\mathrm{ej}} / d a_{*} d t$ to compute the final velocity distributions $h(V, \theta)$ and $h(V, \phi)$. The procedure is similar to that described in the Appendix of Paper II. As the binary shrinks to separation $a<1$, the normalized distribution of ejection velocities for stars with semimajor axis in the interval $a_{*}, a_{*}+d a_{*}$ is

$$
h_{a}\left(V, \theta \mid a_{*}\right)=\frac{1}{\sqrt{a}} h_{1}\left(\frac{V}{\sqrt{a}}, \theta \mid a_{*}\right),
$$

where the ejection speed $V$ was shifted by the factor $1 / \sqrt{a}$ to account for the increase in the circular velocity of the binary $V_{c}$ as the pair shrinks. The prefactor $1 / \sqrt{a}$ normalizes the distribution according to equation (3). The kick velocity function of the expelled population as a whole can then be written as

$h(V, \theta)=\frac{\int_{1}^{a_{f}} \int_{0}^{\infty}\left(d^{2} N_{\mathrm{ej}} / d a_{*} d a\right) h_{a}\left(V, \theta \mid a_{*}\right) d a_{*} d a}{\int_{1}^{a_{f}} \int_{0}^{\infty} \int_{0}^{\pi} \int_{0}^{\infty}\left(d^{2} N_{\mathrm{ej}} / d a_{*} d a\right) h_{a}\left(V, \theta \mid a_{*}\right) d a_{*} d a d \theta d V}$,

where $a_{f}$ is the final binary separation,

$$
\frac{d^{2} N_{\mathrm{ej}}}{d a_{*} d a} \equiv \frac{d^{2} N_{\mathrm{ej}}}{d a_{*} d t} \frac{d t}{d a},
$$

and $d t / d a$ is given by equation (6). The distribution $h_{1}\left(V, \theta \mid a_{*}\right)$ is evaluated for different values of the eccentricity $e$. We can account then for the evolution of binary eccentricity by interpolating the $h_{1}$ distribution on a grid of $e$-values as the orbit decays. The above procedure returns the velocity distribution in units of $V_{c}\left(a_{0}\right)$. The calculation of $h(V, \phi)$ can be performed following the same lines.

Finally, integrating the rate $d^{2} N_{\mathrm{ej}} / d a_{*} d t$ over the entire evolutionary history of the binary yields the quantity $d N_{\mathrm{ej}} / d a_{*}$, which can be compared to $d N / d a_{*}$ to study the depletion of the stellar cusp by three-body interactions. The Monte Carlo technique developed to address this point is described in $\S 5.1$. The functions $d N_{\mathrm{ej}} / d a_{*}$ and $h(V, \theta)$ can be used to check energy conservation, by simply equating the total energy gained by the stars to the increment in the binding energy of the black hole pair, i.e.,

$$
\begin{aligned}
& m_{*} \int_{0}^{\infty} \frac{d N_{\mathrm{ej}}}{d a_{*}} \Phi\left(a_{*}\right) d a_{*} \\
& +\frac{1}{2} m_{*} \int_{0}^{\infty} V^{2} N_{\mathrm{ej}} h(V) d V=\frac{G M_{1} M_{2}}{2}\left(\frac{1}{a_{f}}-\frac{1}{a_{0}}\right),
\end{aligned}
$$

where $\Phi\left(a_{*}\right)=G M_{1} / a_{*}, h(V)=\int h(V, \theta) d \theta$, and $N_{\mathrm{ej}}=\int\left(d N_{\mathrm{ej}} /\right.$ $\left.d a_{*}\right) d a_{*}$. We have checked that energy is conserved to better than $\sim 1 \%$.

It is important to remark at this point that equations (6) and (7) are independent of the absolute value of $M$ and $m_{*}$. Indeed, in equation (6), the total number of interacting stars is $\propto M_{2} / m_{*}$, and the energy exchange term is $\propto M m_{*}$. The scaling factor $1 / M_{1} M_{2}$ cancels out, so that the orbital evolution depends only on $q$ and $e$. The same consideration holds for equation (7): as $\Delta e \propto m_{*} / M$ while the number of interacting stars $\propto M_{2} / m_{*}$, the dependence on $M$ and $m_{*}$ cancels out.

\section{BINARY EVOLUTION}

For comparison with our previous results on the scattering of unbound stars (Paper II), it is convenient to introduce the stellar 
TABLE 1

Binary Shrinking Factors and Final Eccentricities FROM THE Hybrid MODEL

\begin{tabular}{|c|c|c|c|c|c|c|c|}
\hline \multirow[b]{2}{*}{$q$} & \multirow[b]{2}{*}{$e_{0}$} & \multicolumn{2}{|c|}{$\gamma=1.5$} & \multicolumn{2}{|c|}{$\gamma=1.75$} & \multicolumn{2}{|c|}{$\gamma=2$} \\
\hline & & $a_{0} / a_{f}$ & $e_{f}$ & $a_{0} / a_{f}$ & $e_{f}$ & $a_{0} / a_{f}$ & $e_{f}$ \\
\hline \multirow[t]{3}{*}{$1 / 9 \ldots \ldots \ldots . .}$. & 0.1 & 9.63 & 0.608 & 9.89 & 0.350 & 11.38 & 0.179 \\
\hline & 0.5 & 9.99 & 0.972 & 11.55 & 0.907 & 15.77 & 0.753 \\
\hline & 0.9 & 10.06 & 0.998 & 11.80 & 0.992 & 17.73 & 0.969 \\
\hline \multirow[t]{3}{*}{$1 / 27 \ldots \ldots \ldots$} & 0.1 & 8.06 & 0.691 & 8.35 & 0.532 & 10.19 & 0.408 \\
\hline & 0.5 & 8.26 & 0.959 & 9.35 & 0.862 & 12.35 & 0.710 \\
\hline & 0.9 & 8.27 & 0.996 & 9.64 & 0.988 & 14.03 & 0.958 \\
\hline \multirow[t]{3}{*}{$1 / 81 \ldots \ldots \ldots$} & 0.1 & 6.99 & 0.755 & 7.75 & 0.650 & 9.39 & 0.542 \\
\hline & 0.5 & 6.90 & 0.922 & 7.81 & 0.828 & 10.14 & 0.717 \\
\hline & 0.9 & 6.89 & 0.996 & 7.81 & 0.974 & 11.00 & 0.937 \\
\hline \multirow[t]{3}{*}{$1 / 243 \ldots \ldots .}$. & 0.1 & 6.49 & 0.906 & 7.28 & 0.805 & 9.30 & 0.688 \\
\hline & 0.5 & 6.39 & 0.971 & 7.25 & 0.914 & 9.92 & 0.818 \\
\hline & 0.9 & 6.38 & 0.962 & 7.19 & 0.986 & 10.09 & 0.955 \\
\hline \multirow[t]{3}{*}{$1 / 729$} & 0.1 & 6.12 & 0.881 & 6.91 & 0.814 & 8.94 & 0.724 \\
\hline & 0.5 & 5.95 & 0.919 & 6.91 & 0.869 & 9.09 & 0.797 \\
\hline & 0.9 & 5.94 & 0.977 & 6.92 & 0.953 & 9.41 & 0.900 \\
\hline
\end{tabular}

velocity dispersion, $\sigma$, and model the outer stellar component as a singular isothermal sphere (SIS) with density profile

$$
\rho(r)=\frac{\sigma^{2}}{2 \pi G r^{2}} .
$$

We assume that this profile extends inward up to the characteristic radius $r_{0}$ within which the total stellar mass is $2 M_{1}$ (Merritt 2004). Matching the inner cusp described by equation (5) to the outer SIS at $r_{0}$ yields

$$
r_{0}=(3-\gamma) \frac{G M_{1}}{\sigma^{2}}
$$

We also assume that stellar-binary interactions start at separation $a_{0}$ where the enclosed stellar mass is $M_{*}\left(<a_{0}\right)=2 M_{2}$, yielding

$$
a_{0}=\left(\frac{q}{1+q}\right)^{1 /(3-\gamma)}\left[(3-\gamma) \frac{G M}{\sigma^{2}}\right]
$$

This assumption is motivated by recent $N$-body simulations of the hardening of unequal MBHBs in stellar cusps. Matsubayashi et al. (2007) found that dynamical friction is efficient in driving orbital decay only as long as the stellar mass inside the binary semimajor axis is $z M_{2}$. Beyond this point, the evolution of the pair is driven by three-body interactions with individual stars.

\subsection{Orbital Evolution and Eccentricity Growth}

Our results on binary orbital decay are summarized in Table 1. A MBHB can shrink by factors ranging from 6 to 18 depending on $q, e_{0}$, and $\gamma$. The decay factor $a_{0} / a_{f}$ grows with $e_{0}$, as more eccentric binaries can expel stars that are more tightly bound (see Fig. 4). The importance of the initial eccentricity $e_{0}$ is modest for low values of $\gamma$, since in shallow cusps the number of tightly bound stars is small anyway. The factor $a_{0} / a_{f}$ is also a weakly increasing function of $q$; as for higher $q$ the radius of influence of the binary is larger (in terms of $a_{*} / a$; see Fig. 4). The dependence of the decay factor on $\gamma$ simply reflects the fact that for steeper stellar cusps the mean binding energy of the stars is larger. ${ }^{4}$

Binary eccentricity also grows as a function of $q$ and $\gamma$. A shallow cusp increases the relative importance of stars with large $a_{*}$, and the eccentricity growth is then larger. Moreover, binaries with large $q$ are more effective in ejecting stars with $a_{*} / a \ll 1$. As these stars act to circularize the binary orbit, nearly equal mass binaries decay following less eccentric orbits. Figure 8 shows examples of binary evolution as a function of time. Note the different scale of the time axis for the three mass ratios: as shown in Figure 6, it takes nearly equal mass pairs a smaller number of binary orbits to unbind the stellar cusp.

Results for the $\gamma=2$ cusp can also be compared to those obtained in Paper II for the case of a binary interacting with a population of unbound stars. (In Paper II, shrinking factors were normalized to the "hardening radius" $a_{h} \equiv G M_{2} / 4 \sigma^{2}$, where, by definition, $a_{0}=4 a_{h}$ for $\gamma=2$.) Binaries with $e_{0}=0.1$ and $q=$ $(1 / 9,1 / 27,1 / 81,1 / 243)$, for example, shrink by the factors $a_{h} / a_{f}=$ $(2.85,2.55,2.35,2.33)$ according to Table 1 , compared to the corresponding $a_{h} / a_{f}=(2.09,1.49,1.19,1.06)$ for the unbound case; i.e., very unequal mass binaries can only decay by extracting the cusp binding energy.

The evolution of the eccentricity in the bound and unbound cases is compared in Figure 9, for two different initial values of $e_{0}$. Compared to the scattering of unbound stars, the binary eccentricity grows to larger values. This can be understood by the following argument. In the bound case treated here, there are many stars able to extract angular momentum from the binary right from the start. Then for large $a, e$ shows a steep increase. When the orbit shrinks, however, stars with $a_{*}<a$ (increasing circularization rather than eccentricity; see Fig. 5) become more and more relevant for the binary evolution as stars with $a_{*}>a$ get progressively depleted, and as result, $e$ increases at a reduced pace. In the unbound case, the binary interacts with the same distribution of stars independently on its separation. As, in general, significant changes of $e$ happen whenever a strong star-binary interaction occurs, the eccentricity grows more rapidly for small $a$ because stars are slower (in terms of binary circular velocity) and hence more easily captured in "quasi-bound" orbits before ejection. As a matter of fact, the eccentricity growth rate $K$ (see Paper I) increases for $a \ll a_{h}$. In this separation regime, most of the stars interacting with the binary are captured in temporarily bound orbits and would experience a dynamical interaction similar to that of bound stars.

\subsection{Final Coalescence}

Consider now, as in Paper II, the separation at which a MBHB can coalesce in less than $1 \mathrm{Gyr}$ because of the emission of gravitational waves $(\mathrm{GWs})$ :

$$
a_{\mathrm{GW}} \simeq \frac{a_{h}}{250}\left(\frac{1+q}{q}\right)^{3 / 4} M_{1,6}^{1 / 4} F(e)^{1 / 4}
$$

\footnotetext{
${ }^{4}$ Note that we have not included the effect of "returning stars" on the decay of the binary. These are ejected stars that do not escape the host bulge, return on small impact parameter orbits, and can have a secondary superelastic interaction with the MBHB. The role of secondary slingshots was analyzed in Paper II, where it was found that they can boost orbital decay by as much as a factor of 2 for nearly equal mass binaries, but do not contribute significantly (less than $20 \%$ for $q=1 / 9$ and less than $5 \%$ for $q=1 / 81$; see Table 1 of Paper II) to binary hardening for very unequal mass pairs. In the bound case, the effect of returning stars is likely to be even smaller. Consider for example the case of an isothermal stellar profile: the impact of secondary slingshots is proportional to the number of stars that can interact with the MBHB more than once, i.e., to the size of the loss cone after the first interaction. For bound stars this is at least a factor of $\sim 2$ smaller than in the unbound case, so even for $q=1 / 9$, returning stars would cause at most a $\sim 10 \%$ increase in binary hardening.
} 


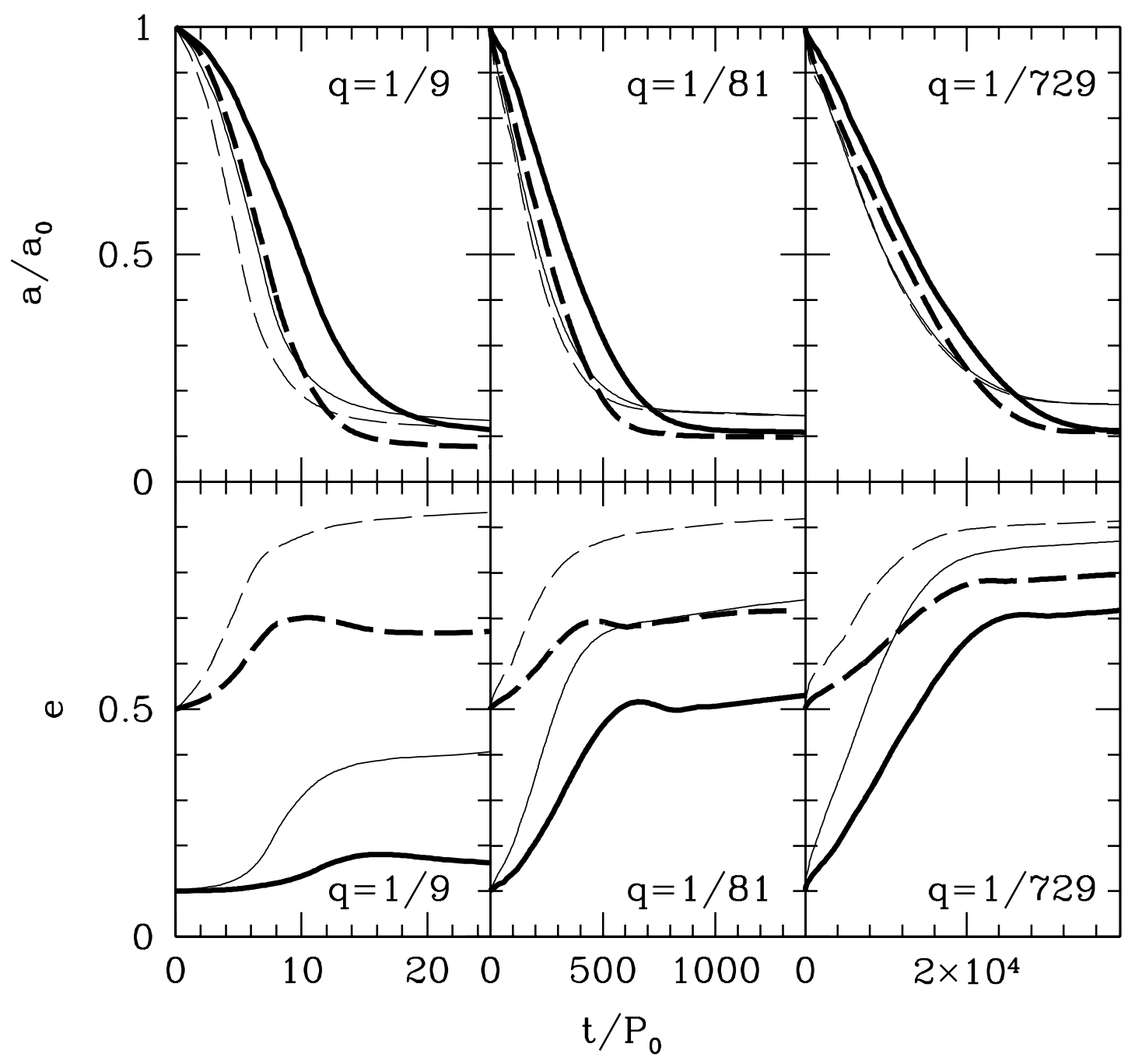

FIG. 8. - Time evolution of the binary semimajor axis (top) and eccentricity (bottom) for $q=1 / 9$ (left), $q=1 / 81$ (middle), and $q=1 / 729$ (right). In all panels the thick lines are for a $\gamma=2$ stellar cusp, while the thin lines are for $\gamma=1.5$. The initial binary eccentricity is assumed to be 0.1 (solid lines) and 0.5 (dashed lines). 


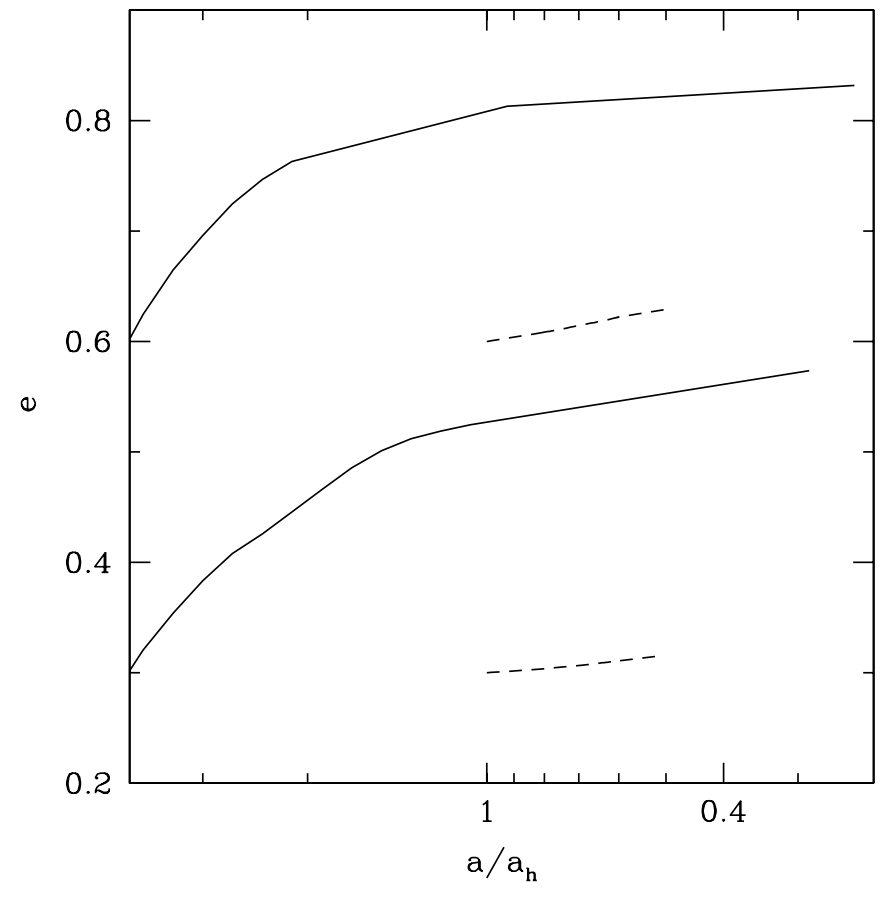

FIG. 9.-Evolution of binary eccentricity as a function of orbital separation, for the case of interactions with unbound stars (Papers I and II, dashed lines) and with a bound cusp (solid lines). The binary is embedded in an SIS, its mass ratio is $q=1 / 9$, and its initial eccentricity is $e_{0}=0.3$ and 0.6 . Note that the scattering experiments with unbound stars start at $a=a_{h}$, while those with bound stars start at $a=4 a_{h}$ (see text for details). In the unbound case the evolution of eccentricity is negligible ( $K \approx 0$; Sesana et al. 2006) for $a>a_{h}$.

where $M_{1,6} \equiv M_{1} / 10^{6} M_{\odot}$ and, to fourth order in $e$ (Peters 1964),

$$
F(e) \equiv\left(1-e^{2}\right)^{-7 / 2}\left(1+\frac{73}{24} e^{2}+\frac{37}{96} e^{4}\right) .
$$

Assuming as before that three-body interactions begin operating at separation $a_{0}$, and combining equations (22) and (23), we get

$$
\frac{a_{f}}{a_{\mathrm{GW}}} \simeq 10^{3}\left(\frac{a_{0}}{a_{f}}\right)^{-1}(3-\gamma)\left(\frac{q}{1+q}\right)^{(1+\gamma) /(12-4 \gamma)} M_{1,6}^{-1 / 4} F(e)^{-1 / 4} .
$$

If $a_{f} / a_{\mathrm{GW}}<1$, then a MBHB would coalesce in less than $1 \mathrm{Gyr}$ after interaction with a bound stellar cusp. As shown in the "coalescence diagram" of Figure 10, eccentric, massive, very unequal mass binaries embedded in steep stellar cusps are favored to reach coalescence. The steeper the cusp, the wider the portion of the $\left(M_{1}, q\right)$-plane where coalescence can be reached within $1 \mathrm{Gyr}$.

\section{EVOLUTION OF THE STELLAR CUSP}

\subsection{Cusp Erosion}

The orbital decay of the pair occurs following the ejection of a mass $\sim(2-4) M_{2}$ (depending on cusp slope and binary eccentricity) and results in the progressive erosion of the stellar cusp. Eccentric binaries can shrink rapidly by scattering at pericenter a fewer number of deeply bound stars. More mass is expelled in the case of shallower cusps, because of the larger number of stars surrounding the binary just outside $a_{0}$. Our hybrid approach allows us to compute the binary-driven evolution of the stellar density profile, once isotropy is assumed. At this stage, we do not perform a self-consistent treatment of the evolution of the anisotropy of the stellar cusp. Self-consistent integration of the orbital decay yields $d N_{\mathrm{ej}} / d a_{*}$, and the number of stars that remain bound to $M_{1}$ as a function of $a_{*}$ is simply

$$
\frac{d N_{\mathrm{bd}}}{d a_{*}}=\frac{d N_{*}}{d a_{*}}-\frac{d N_{\mathrm{ej}}}{d a_{*}} .
$$

We assume that the stellar cusp remains isotropic, i.e., $\left\langle e_{*}\right\rangle=0.667$. The $a_{*}$ domain is then subdivided in intervals $\Delta a_{*}$, and for each interval a number of bound stars $\propto\left(d N_{\mathrm{bd}} / d a_{*}\right) \Delta a_{*}$ are generated with angular momentum distribution $\propto L_{*}^{2}$. We then compute the probability of finding a star at a distance between $r$ and $r+d r$ from $M_{1}$ (this is proportional to the time spent at such distance along its orbit) and reconstruct the stellar density profile $\rho(r)$. Figure 11 shows the profile before and after binary shrinking. The cusp is eroded between $\sim 0.01 a_{0}$ and $\sim 2 a_{0}$, depending on $e_{0}$. For $r \lesssim a_{0}$, an SIS is flattened to $\rho \propto r^{-0.7}$, while a $r^{-1.5}$ cusp becomes $\rho(r) \propto r^{-0.5}$. Such results are independent of the mass ratio $q$.

As three-body interactions and stellar ejections tend to circularize the orbits of ambient stars, one may wonder about the validity of the assumption that the cusp remains isotropic. We checked that even assuming that all stars still bound to the binary at the end of the process were set on circular orbits (i.e., maximum tangential anisotropy), then the cusp slope would be flattened at most by a factor of $\simeq 0.3$ (for $\gamma=2$ and $q=1 / 729$ ) with respect to the isotropic case. Differences in the cusp slope $\lesssim 0.1$ are common for initially shallower cusps and/or larger mass ratios. We conclude that depending on $\gamma$ and on the final anisotropy of the bound stars, the slingshot mechanism creates a central core as flat as $\rho(r) \propto$ $r^{-0.4}$ to $r^{-0.8}$.

\subsection{Distribution of Kick Velocities}

The distribution of stellar ejection velocities in units of the binary circular velocity at the initial separation $a_{0}, V_{c, 0}$, is shown in Figure 12. The distribution cannot be fit by a single or a broken power law, as in the case of the kick velocities imparted at fixed binary given separation, as the derivative of the distribution is a monotonic decreasing function of the ejection speed. While the peak of the distribution shifts toward smaller $V / V_{c, 0}$ values as $q$ decreases $\left(V_{\text {peak }} \propto \sqrt{q}\right)$, the high-velocity tail is independent of $q$. For initially eccentric binaries, the velocity of $M_{2}$ at periastron is $>V_{c, 0}$, and a significant number of HVSs can then be generated. Note that the speed distribution is only weakly dependent on $\gamma$ once $V$ is expressed in units of $V_{c, 0}$.

\subsection{Ejected Mass}

Integration of the kick velocity distribution gives the ejected mass $M_{\mathrm{ej}}$. This quantity is plotted in Figure 13 as a function of $q$ for different lower velocity thresholds. As discussed above, the total number of ejected stars is approximately $\propto q$ and is weakly dependent on $e_{0}$ and $\gamma$. Stellar ejection occurs in a burst lasting from few tens to several thousands of binary orbital periods (see Fig. 14, note the different scale of the time axis in the four panels), i.e., from $10^{5}$ to $10^{7} \mathrm{yr}$. The highest velocity stars are delayed with respect to the bulk of ejections, as large kicks require close binary separations. The ejection rate peaks at earlier times in the case of more eccentric binaries and is larger for steeper cusps.

It must be pointed out that the quantity $M_{\mathrm{ej}}$ plotted in Figure 13 accounts only for the mass ejected by energetic three-body interactions occurring in the final stage of the binary evolution. During the entire binary evolution, stars in galaxy nuclei are also displaced by the heating associated with dynamical friction - the cumulative effect of many weak encounters with distant stars. According to Merritt (2006b), the total mass displaced by dynamical friction 


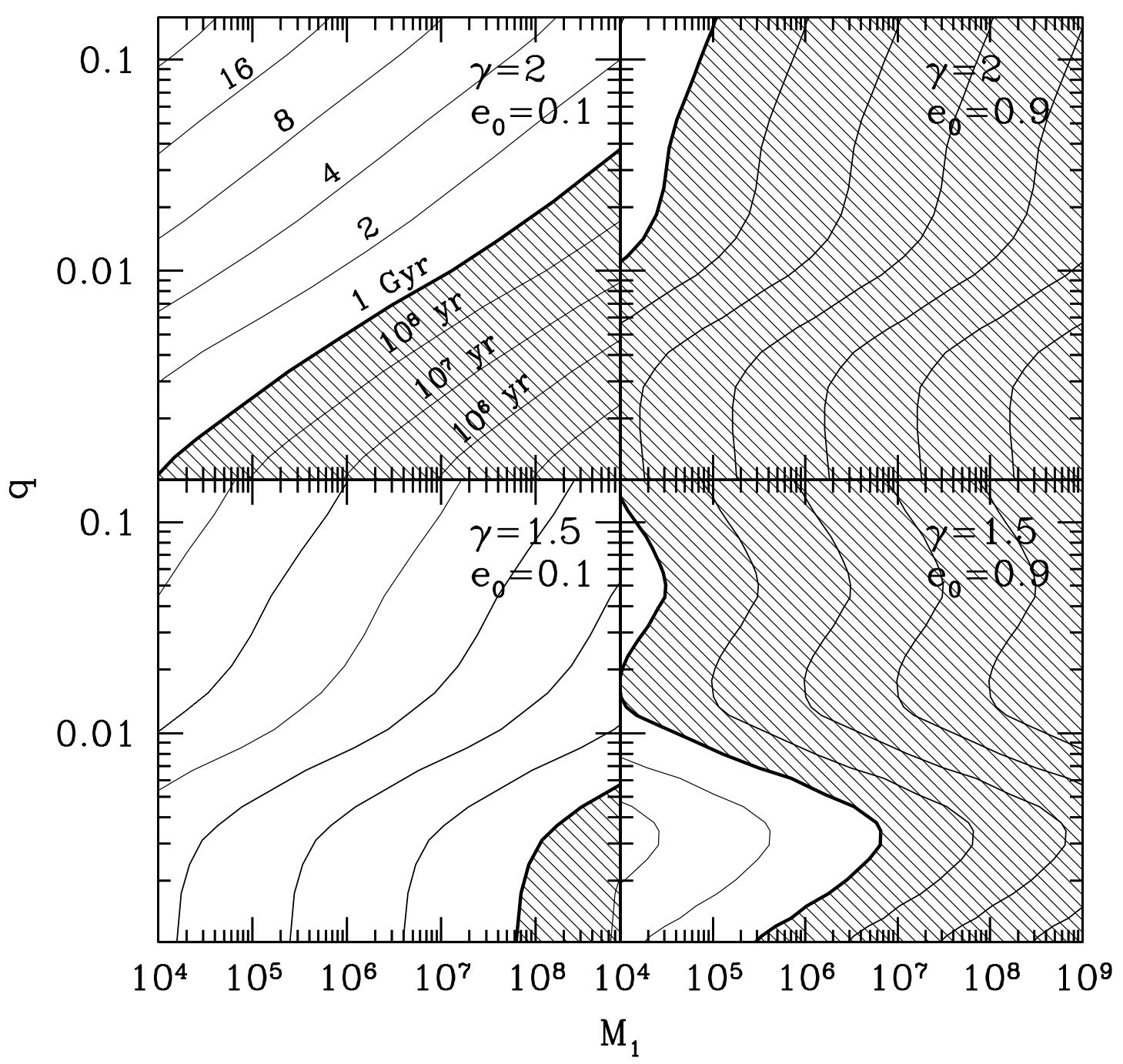

FIG. 10.-Coalescence diagram. In each panel, the thick line delimits those MBHBs that can coalesce, because of GW emission, in less than 1 Gyr starting from separation $a_{f}$ (shaded area). As shown in the top left panel, level curves to the left of that line (thick curve) are labeled according to the ratio $a_{f} / a_{\mathrm{GW}}$ reached by the binary at the end of the shrinking process (eq. [25]), while level curves to the right are labeled according to the coalescence time at $a_{f}$. 


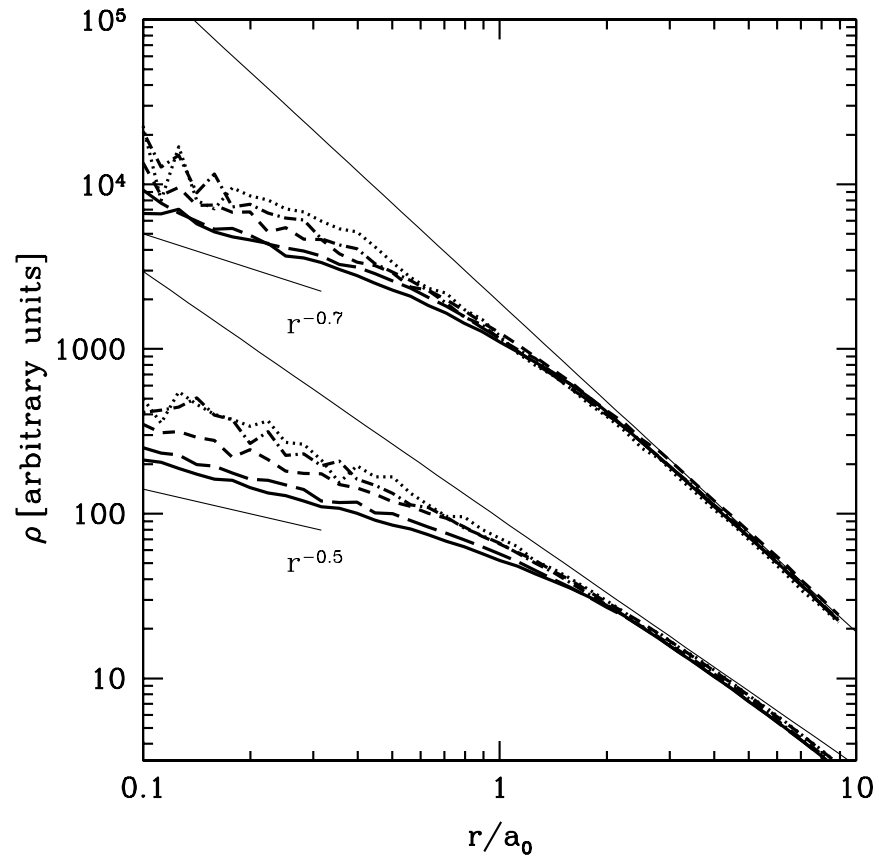

FIG. 11.-Cusp stellar density profiles (in arbitrary units) before (thin lines) and after (thick lines) erosion by the shrinking binary for $q=1 / 9$ (solid line), $q=$ $1 / 27$ (long-dashed line) $q=1 / 81$ (short-dashed line) $q=1 / 243$ (dot-dashed line) and $q=1 / 729$ (dotted line). The upper (lower) set of curves is for $\gamma=2(\gamma=1.5)$. Fiducial $r^{-0.7}$ and $r^{-0.5}$ power laws are shown for reference.

depends only weakly on binary mass ratio (for $q$ in the range $0.025<q<0.5)$ and has little or no effect on the innermost stellar cusp for $q<0.1$ (see Fig. 5 in Merritt 2006b). While the total mass deficit accumulated during binary evolution appears to scale with the mass of the binary (i.e., to $M_{1}$ for small $q$ ), the mass ejected after the energetic encounters discussed in this paper, and therefore the number of expected HVSs, scales with the mass $M_{2}$ of the intruder. The number of stars ejected above a given velocity threshold (in units of $V_{c, 0}$ ) is also a weak function of $e_{0}$ and $\gamma$.

\subsection{Angular Properties of HVSs}

The angular properties of HVSs show several peculiar features, qualitatively similar to those discussed in Paper I for the scattering of an unbound stellar population. The ejected stars are flattened in the binary orbital plane and, in the case of eccentric binaries, are grouped into a "broad jet" aligned to the velocity of $M_{2}$ at periastron. Both anisotropies are more pronounced in stars undergoing a stronger interaction and receiving larger kicks. The anisotropy of the HVS population can be quantified by computing as a function of binary separation the angles $\left\langle\theta^{2}\right\rangle$ and $\langle\phi\rangle$, where $-\pi / 2<\theta<\pi / 2$ is the latitude of the star (the angle between the velocity vector $V$ at infinity and the binary orbital plane) and $0<$ $\phi<2 \pi$ is its longitude (the angle between the projection onto the binary orbital plane of the velocity vector at infinity and the $x$-axis). For a spherically symmetric distribution, $\left\langle\theta^{2}\right\rangle \simeq 0.47$ and $\langle\phi\rangle=\pi$. We find $\left\langle\theta^{2}\right\rangle \sim 0.35-0.40$ for all HVSs. As a general trend, stars ejected above a given speed tend to become more isotropic as the binary shrinks, confirming the analytical result of Levin (2006). This effect was already seen in three-body scattering experiments of unbound stars (see Paper I for a detailed discussion) and is related to the fact that as the pair decays, its circular velocity grows, and even stars experiencing relatively weak encounters can attain large kick velocities. In terms of longitude, HVSs are ejected almost isotropically, although an azimuthal anisotropy becomes apparent with increasing kick velocities. The highvelocity tail of the distribution is formed by stars expelled after a close encounter with $M_{2}$ near its periastron, in a broad jet with $\langle\phi\rangle \simeq 3 / 2 \pi$. As the binary potential is non-Keplerian, such a broad jet will precess during binary evolution on a timescale that depends on $q$ and $\gamma$ (Levin 2006).

\section{THE CASE OF SGR A*}

Most of the results presented in the previous sections are scale invariant; i.e., they are independent on the absolute value of $M$ and on the chosen normalization of the stellar cusp. The only underlying assumption is that the interaction between the MBHB and ambient stars begins when the stellar mass inside the binary orbit is equal to $2 M_{2}$. We are interested in scaling our results to the scattering of stars bound to Sgr $\mathrm{A}^{*}$, the massive black hole in the Galactic center, by an inspiraling companion of intermediate mass ( Yu \& Tremaine 2003; Sesana et al. 2007b). Let us first express equations (20), (21), and (22) in physical units:

$$
\begin{gathered}
r_{0}=(0.43 \mathrm{pc})(3-\gamma) M_{1,6} \sigma_{100}^{-2}, \\
\rho_{0}=\left(1.96 \times 10^{6} M_{\odot} \mathrm{pc}^{-3}\right)(3-\gamma)^{-2} M_{1,6}^{-2} \sigma_{100}^{6}, \\
a_{0}=(0.43 \mathrm{pc})(3-\gamma) M_{1,6} \sigma_{100}^{-2} q^{1 /(3-\gamma)}(1+q)^{(2-\gamma) /(3-\gamma)},
\end{gathered}
$$

where $\sigma_{100}$ is measured in units of $100 \mathrm{~km} \mathrm{~s}^{-1}$. From $a_{0}$, the time unit of our experiments is then

$$
P_{0}=\left(1.3 \times 10^{5} \mathrm{yr}\right)(3-\gamma)^{3 / 2} M_{1,6}^{1 / 4} q\left(\frac{q}{1+q}\right)^{(2 \gamma-3) /(6-2 \gamma)} .
$$

The stellar density profile around the Galactic center can be described as a double power law, with outer slope $\simeq-2$ and inner slope $\simeq-1.5$ (Genzel et al. 2003; Schodel et al. 2007). The massive black hole Sgr A* weighs $\simeq 3.5 \times 10^{6} M_{\odot}$ (Schodel et al. 2002; Ghez et al. 2005). Using $M_{1,6}=3.5, \sigma_{100}=1$, and $\gamma=1.5$, from equations (27) and (28) we obtain $r_{0}=2.26 \mathrm{pc}$ and $\rho_{0}=$ $7 \times 10^{4} M_{\odot} \mathrm{pc}^{-3}$, in good agreement with the most recent observations (Merritt 2006a; Schodel et al. 2007). For the relevant values of $q$ and $\gamma$, the typical timescale for orbital decay ranges between $\sim 10^{5}$ and $\sim 10^{7} \mathrm{yr}$ (see eq. [30]). In the following, we will consider two different mass ratios for the putative MBHB at the Galactic center, $q=1 / 243$ and $1 / 729$, corresponding to an inspiraling IMBH of mass $M_{2} \simeq 1.4 \times 10^{4} M_{\odot}$ and $M_{2} \simeq 4.8 \times$ $10^{3} M_{\odot}$, respectively, as well as two different slopes for the initial inner stellar cusp, $\gamma=1.5$ and 1.75 . We will also study the impact of binary initial eccentricity. The parameters of the different models considered are listed in Table 2.

The dynamical evolution of a putative IMBH-Sgr A* binary is displayed in Figure 15 for $e_{0}=0.1$. The two top panels show the time-changing semimajor axis $a$ and eccentricity $e$. The former shrinks to $10^{-2}$ to $10^{-3} \mathrm{pc}$ in 2-15 Myr, depending on $q$ and $\gamma$. Lighter IMBHs reach smaller separations on a longer timescale. Our results for the case $\gamma=1.75$ can be directly compared to the numerical simulations of Baumgardt et al. (2006) and Matsubayashi et al. (2007) and are found to be in excellent agreement. The eccentricity increases rapidly in all cases to values $\gtrsim 0.8$. We have checked that when $e_{0}>0.3$, the binary eccentricity can reach values as large as $e \gtrsim 0.95$. This is again in agreement with the results of Baumgardt et al. (2006) and Matsubayashi et al. (2007). In Figure 15 we have marked with a horizontal dotted line the separation at which the binary can coalesce in 1 Gyr because of GW emission (eq. [23]). Only for a cusp as steep as $\gamma=1.75$ does 


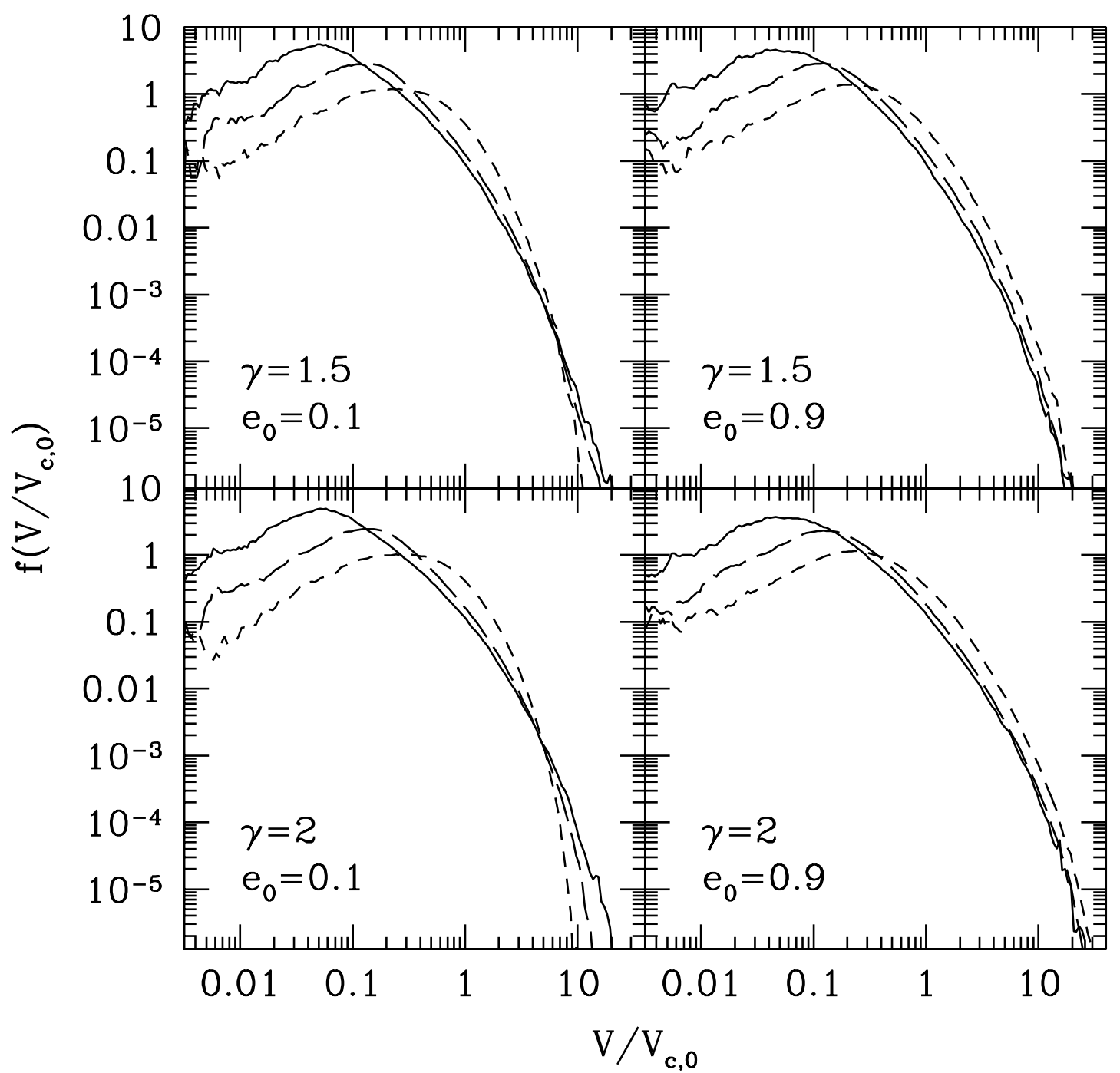

FIG. 12.-Final velocity distribution of ejected stars as a function of $V / V_{c, 0}$ for $q=1 / 9$ (short-dashed lines), $q=1 / 81$ (long-dashed lines), and $q=1 / 729$ (solid lines). The assumed values of $\gamma$ and $e_{0}$ are listed in each panel. 


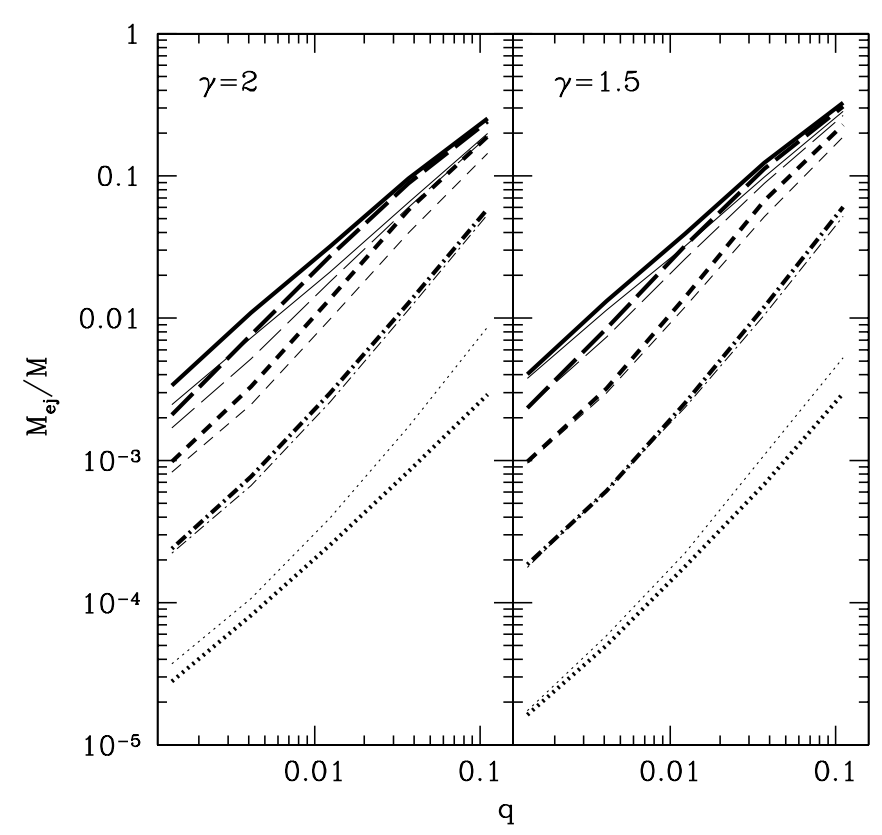

FIG. 13.- Mass ejected from the binary, scaled to the total binary mass, as a function of $q$, for $\gamma=2$ (left) and $\gamma=1.5$ (right). Thick lines and thin lines denote $e_{0}=0.1$ and 0.9 , respectively. In each set of curves the lines refer, from top to bottom, to all stars with kick velocities $V>0$ (the total mass expelled by the binary) and with $\log \left(V / V_{c, 0}\right)>-1+0.5 n$ for $n=0,1,2$, and 3 .

the pair actually reach such separation. One should note that while in our hybrid model the eccentricity evolves smoothly, in a realistic situation it will undergo discontinuous "jumps" triggered by rare close encounters, which could induce extreme eccentricities and accelerate coalescence. The two bottom panels of Figure 15 show the resulting stellar density profiles after binary erosion. Cusps are flattened to $\rho \propto r^{-0.7}$ in the central few $\times 10^{-2} \mathrm{pc}$. The numerical simulations of Baumgardt et al. (2006) and Matsubayashi et al. (2007) produce somewhat shallower slopes, a discrepancy that may be associated with our assumption of an isotropic stellar cusp after the interaction.

The heating of the cusp results in the creation of a population of HVSs. This is of particular interest since the discovery of the first HVS in the Milky Way (Brown et al. 2005), and an IMBH inspiral onto $\mathrm{Sgr} \mathrm{A}^{*}$ is regarded as a possible source of hypervelocity ejections (although the tidal breakup of close binaries by Sgr A* seems to be supported by observations and statistical studies; see, e.g., Perets 2007; Sesana et al. 2007b). In Figure 16 we plot the stellar ejection rates as a function of time for different models and for different velocities at the radius of influence of Sgr A*. Modeling the Milky Way potential as the sum of a luminous component (Miyamoto \& Nagai 1975) and a dark matter halo (Widrow \& Dubinski 2005), we find an escape velocity from the Milky Way of $\simeq 840 \mathrm{~km} \mathrm{~s}^{-1}$ at $r_{\text {inf }}$. This velocity threshold translates, in such a gravitational potential, into about $450 \mathrm{~km} \mathrm{~s}^{-1}$ $10 \mathrm{kpc}$ away from Sgr A*. Stars with $V>300 \mathrm{~km} \mathrm{~s}^{-1}$ at $r_{\text {inf }}$ do not leave the bulge, while stars with $V>600 \mathrm{~km} \mathrm{~s}^{-1}$ can reach $4 \mathrm{kpc}$ away from the Galactic center. Stars with $V>900$ and $>1200 \mathrm{~km} \mathrm{~s}^{-1}$ are not bound to the Milky Way and, at a reference distance of $10 \mathrm{kpc}$, have still velocities of 600 and $1000 \mathrm{~km} \mathrm{~s}^{-1}$, respectively. Figure 16 shows that the ejection occurs in a relatively short burst lasting a few million years, with higher velocity stars being produced at increasingly later times. At peak, the ejection rate of HVSs with $V>900 \mathrm{~km} \mathrm{~s}^{-1}$ varies between $5 \times 10^{-5}$ and $2 \times 10^{-3} \mathrm{yr}^{-1}$, depending on $q$ and $\gamma$. The rate is larger in steeper cusps, as stars are more centrally concentrated and are

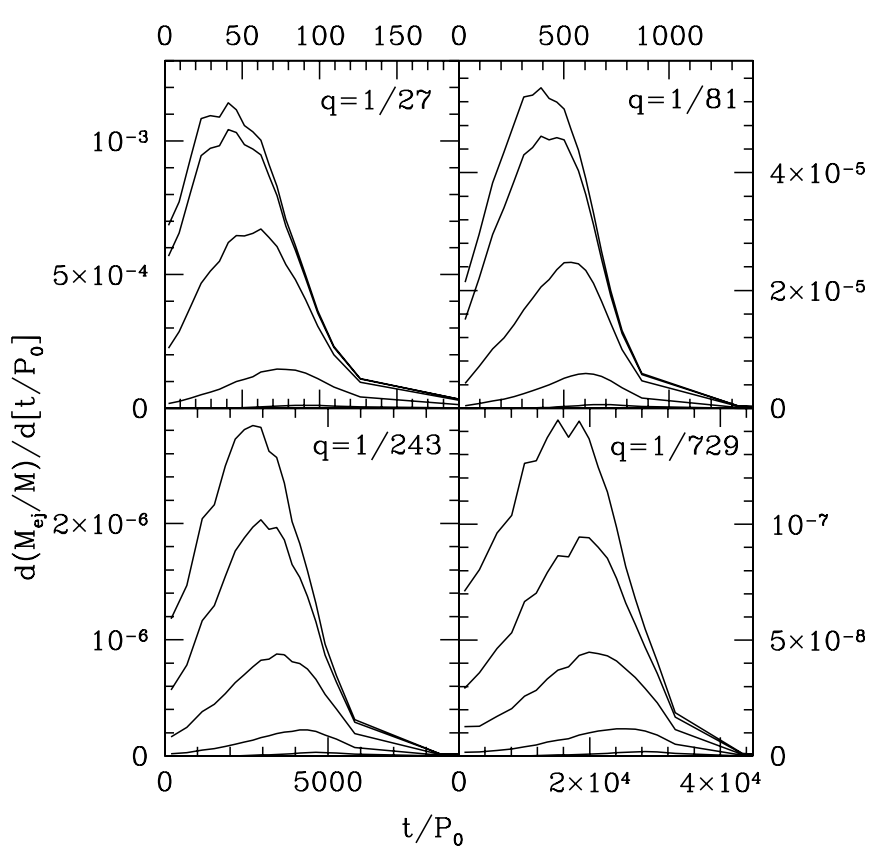

FIG. 14. - Mass ejection rate for binaries with $e_{0}=0.1$ and different mass ratios embedded in an SIS. Lines refer, from top to bottom, to all stars with kick velocities $V>0$ (the total mass expelled by the binary) and with $\log \left(V / V_{c, 0}\right)>-1+0.5 n$ for $n=0,1,2$, and 3 . Note that the time axis has a different scale in each panel.

scattered when the binary separation is smaller and orbital velocity larger.

The speed distribution of HVSs depends on the details of the model. In the range $300-1000 \mathrm{~km} \mathrm{~s}^{-1}$ (the velocity range of the HVSs observed by Brown et al. 2006), and at a galactocentric distance of $55 \mathrm{kpc}$ (the average distance of the observed HVSs), the distribution can be approximated by a power law $f(V) \propto V^{-1.5}$, almost independent of $q, e_{0}$, and $\gamma$. Assuming $m_{*}=1 M_{\odot}$, we find a predicted number of HVSs with $V>840 \mathrm{~km} \mathrm{~s}^{-1}$ at $r_{\mathrm{inf}} N_{\mathrm{HVS}} \simeq$ 525 (1290) for $q=1 / 729$ and $\gamma=1.5$ (1.75). This number roughly doubles for $q=1 / 243$ and is fairly independent on $e_{0}$. Our results are consistent with Baumgardt et al. (2006), who estimate $N_{\mathrm{HVS}} \sim$ 1700 for $M_{2}=10^{4} M_{\odot}$ and $N_{\mathrm{HVS}} \sim 900$ for $M_{2}=3 \times 10^{3} M_{\odot}$. A peak of ejection occurs after $\sim 1-2$ Myr. Levin (2006) finds a comparable numbers of stars expelled and a similarly peaked ejection rate.

\section{SUMMARY}

We have performed, for the first time, scattering experiments between a MBHB and stars drawn from a cusp bound to the primary hole. We have studied the dynamics of the pair and its orbital decay by three-body interactions, the impact of the gravitational slingshot on the stellar density profile, and the properties of the ejected stellar population, and we have scaled our results to the case of Sgr $\mathrm{A}^{*}$. Our results can be quickly summarized as follows.

1. The extraction of the cusp binding energy causes the binary to shrink by a larger factor compared to the scattering of unbound stars. The effect is more noticeable in the case of small mass ratios $q$.

2. The binary orbital eccentricity increases much more rapidly compared to the unbound case. The eccentricity growth is more pronounced in small mass ratio binaries and for shallower stellar cusps.

3. The combined effects of enhanced orbital decay and eccentricity growth lead very unequal mass binaries to the gravitational 
TABLE 2

Parameters of Two Different Models for the Stellar Cusp at the Galactic Center

\begin{tabular}{rccccrr}
\hline \hline$\gamma$ & $\begin{array}{c}r_{0} \\
(\mathrm{pc})\end{array}$ & $\begin{array}{c}\rho_{0} \\
\left(M_{\odot} \mathrm{pc}^{-3}\right)\end{array}$ & $q$ & $\begin{array}{c}a_{0} \\
(\mathrm{pc})\end{array}$ & $\begin{array}{c}T_{0} \\
(\mathrm{yr})\end{array}$ & $\begin{array}{r}V_{c, 0} \\
\left(\mathrm{~km} \mathrm{~s}^{-1}\right)\end{array}$ \\
\hline $1.5 \ldots \ldots \ldots \ldots \ldots \ldots \ldots$ & 2.25 & $7.1 \times 10^{4}$ & $1 / 243$ & $5.8 \times 10^{-2}$ & 1344 & 510 \\
& 2.25 & $7.1 \times 10^{4}$ & $1 / 729$ & $2.8 \times 10^{-2}$ & 448 & 735 \\
\hline $1.75 \ldots \ldots \ldots \ldots \ldots \ldots \ldots$ & $10^{5}$ & $1 / 243$ & $2.3 \times 10^{-2}$ & 340 & 806 \\
& 1.88 & $10^{5}$ & $1 / 729$ & $9.6 \times 10^{-3}$ & 91 & 1250 \\
\hline
\end{tabular}

Notes.-The quantities $\gamma, r_{0}, \rho_{0}, q, a_{0}, T_{0}$, and $V_{c, 0}$ are, respectively, the cusp slope, the cusp characteristic radius, the density at $r_{0}$, the binary mass ratio, the binary separation at which gravitational slingshots start, the binary orbital period at $a_{0}$, and the binary circular velocity at $a_{0}$.

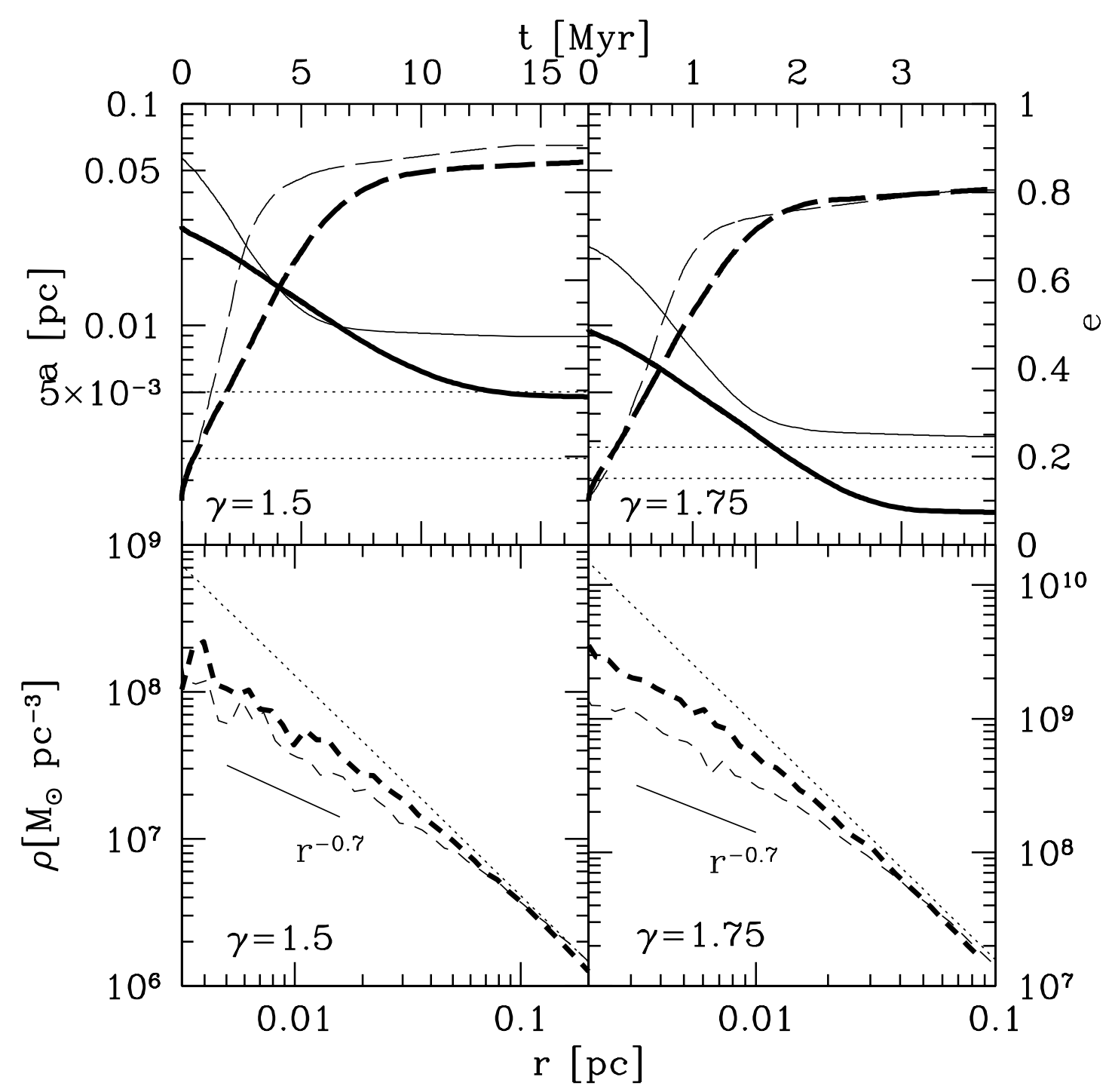

FIg. 15.-Case of Sgr A*. Top: Time evolution of binary semimajor axis (solid lines, left axis scale) and eccentricity (dashed lines, right axis scale) for $e_{0}=0.1$. The dotted lines mark the separation at which binaries can coalesce because of GW emission in <1 Gyr. Bottom: Evolution of the stellar density profile. In all panels, the thin lines are for $q=1 / 243$, the thick lines for $q=1 / 729$. 


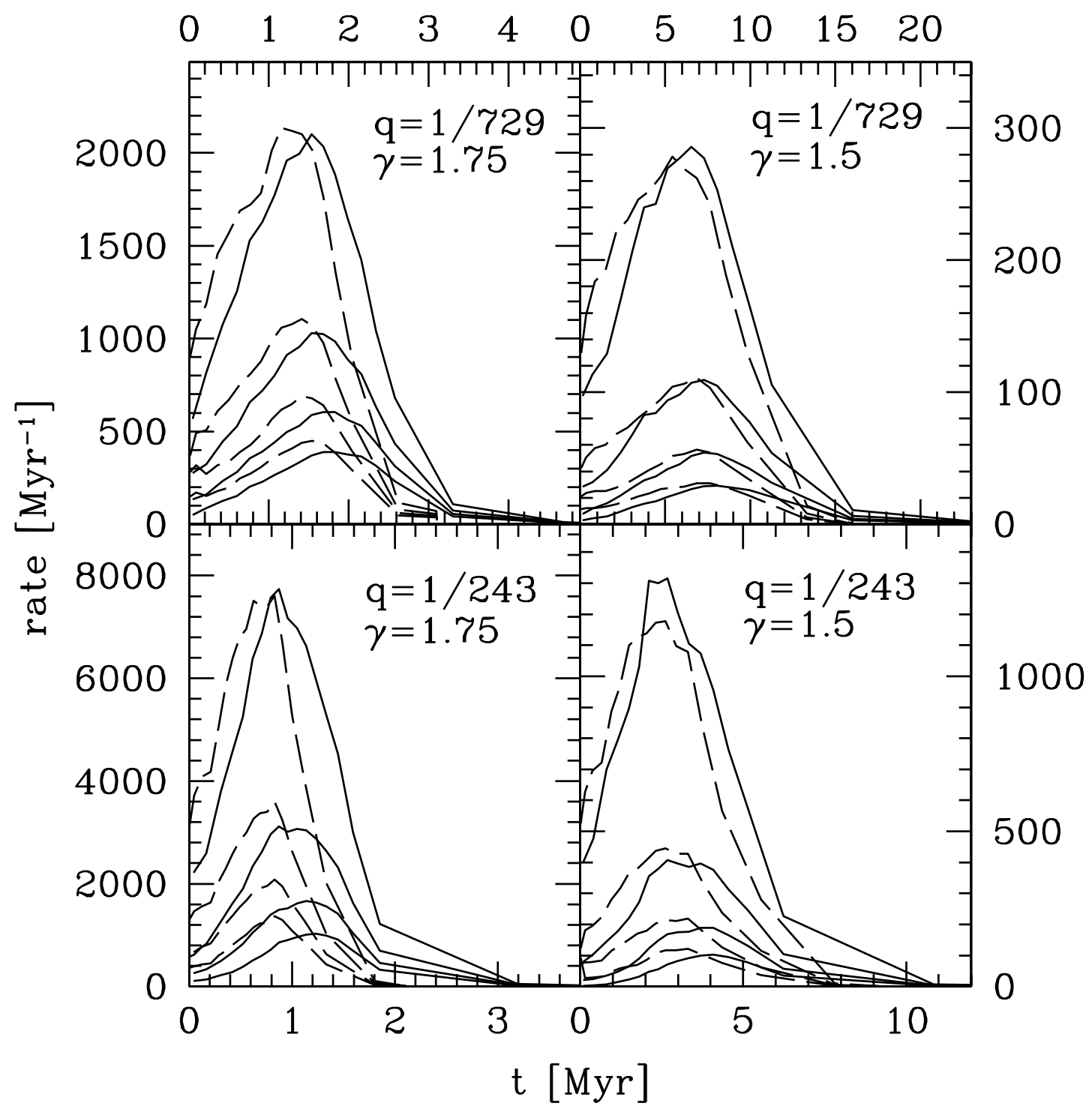

FIG. 16.-Ejection rate of HVSs for an IMBH falling onto Sgr $\mathrm{A}^{*}$ for $e_{0}=0.1$ (solid lines) and $e_{0}=0.9$ (dashed lines). For each set of curves, lines from top to bottom correspond to a velocity threshold $V>300,600,900$, and $1200 \mathrm{~km} \mathrm{~s}^{-1}$ at the radius of influence of Sgr A*, respectively.

wave coalescence phase. The detailed fate of the pair depends on the absolute value of its mass. More massive binaries decay faster.

4. The stellar cusp is eroded, and the total mass removed by strong three-body encounters is $2-4$ times the mass of the secondary hole. While the mass deficit caused by dynamical friction in a merger event scales with the binary mass (and involves mostly distant stars), the mass of stars ejected from the inner cusp by highly energetic interactions scales with $M_{2}$. Ejection occurs in a "burst" lasting from few tenths to several thousands of binary orbital periods, depending on $q$.

5. Scaled to the scattering of stars bound to Sgr A* by an inspiraling IMBH, our results imply the formation of a core of $0.1 \mathrm{pc}$ in $1-10 \mathrm{Myr}$, as well as the ejection of 500-2500 HVSs moving with speeds sufficient to escape the gravitational field of the Milky Way. In Sesana et al. (2007b) we used the Brown et al.
(2007) sample of unbound and bound HVSs together with numerical simulations of the propagation of HVSs in the Milky Way halo to constrain this ejection mechanism, and we have shown that it appears to produce a spectrum of ejection velocities that is too flat compared to the observations. Future astrometric (e.g., Gaia) and deep wide-field (e.g., LSST) surveys should unambiguously identify the ejection mechanism of HVSs and probe the Milky Way potential on scales as large as $200 \mathrm{kpc}$ (Gnedin et al. 2005; Yu \& Madau 2007).

Support for this work was provided by NASA grant NNG04GK85G (P. M.).

\section{REFERENCES}

Baumgardt, H., Gualandris, A., \& Portegies Zwart, S. 2006, MNRAS, 372, 174 Begelman, M. C., Blandford, R. D., \& Rees, M. J. 1980, Nature, 287, 307 Brown, W. R., Geller, M. J., Kenyon, S. J., \& Kurtz, M. J. 2005, ApJ, 622, L33 2006, ApJ, 647, 303

Brown, W. R., Geller, M. J., Kenyon, S. J., Kurtz, M. J., \& Bromley, B. C. 2007, ApJ, 660, 311
Dormand, J. R., \& Prince, P. J. 1978, Celest. Mech., 18, 223

Ebisuzaki, T., Makino, J., \& Okumura, S. K. 1991, Nature, 354, 212

Faber, S. M., et al. 1997, AJ, 114, 1771

Genzel, R., et al. 2003, ApJ, 594, 812

Ghez, A. M., et al. 2005, ApJ, 620, 744

Gnedin, O. Y., Gould, A., Miralda-Escudé, J., \& Zentner, A. R. 2005, ApJ, 634, 344 
Graham, A. W., \& Guzmán, R. 2003, AJ, 125, 2936

Hairer, E., Norsett, S. P., \& Wanner, G. 1993, Solving Ordinary Differential Equations I: Nonstiff Problems (Berlin: Springer)

Ivanov, P. B., Polnarev, A. G., \& Saha, P. 2005, MNRAS, 358, 1361

Levin, Y. 2006, ApJ, 653, 1203

Makino, J., \& Ebisuzaki, T. 1996, ApJ, 465, 527

Matsubayashi, T., Makino, J., \& Ebisuzaki, T. 2007, ApJ, 656, 879

Mayer, L., Kazantzidis, S., Madau, P., Colpi, M., Quinn, T., \& Wadsley, J. 2007, Science, 316, 1874

Merritt, D. 2004, in Carnegie Observatories Centennial Symposia: Coevolution of Black Holes and Galaxies, ed. L. C. H. (Cambridge: Cambridge Univ. Press), 263

Merritt, D. 2006a, Rep. Prog. Phys., 69, 2513 2006b, ApJ, 648, 976

Milosavljevic, M., \& Merritt, D. 2001, ApJ, 563, 34

Miyamoto, M., \& Nagai, R. 1975, PASJ, 27, 533

Perets, H. B. 2007, preprint (arXiv:0712.1888)
Peters, P. C. 1964, Phys. Rev. B, 136, 1224

Quinlan, G. D. 1996, NewA, 1, 35

Quinlan, G. D., \& Hernquist, L. 1997, NewA, 2, 533

Quinlan, G. D., Hernquist, L., \& Sigurdsson, S. 1995, ApJ, 440, 554

Ravindranath, S., Ho, L. C., Peng, C. Y., Filippenko, A. V., \& Sargent, W. L. W. 2001, AJ, 122, 653

Schodel, R., et al. 2002, Nature, 419, 694 2007, A\&A, 469, 125

Sesana, A., Haardt, F., \& Madau, P. 2006, ApJ, 651, 392 (Paper I) 2007a, ApJ, 660, 546 (Paper II) 2007b, MNRAS, 379, L45

Volonteri, M., Haardt, F., \& Madau, P. 2003a, ApJ, 582, 559

Volonteri, M., Madau, P., \& Haardt, F. 2003b, ApJ, 593, 661

Widrow, L. M., \& Dubinski, J. 2005, ApJ, 631, 838

Yu, Q., \& Madau, P. 2007, MNRAS, 379, 1293

Yu, Q., \& Tremaine, S. 2003, ApJ, 599, 1129 